\title{
Rippling: a heuristic for guiding inductive proofs
}

\author{
Alan Bundy, Andrew Stevens*, Frank van Harmelen **, \\ Andrew Ireland and Alan Smaill \\ Department of Artificial Intelligence, University of Edinburgh, 80 South Bridge, \\ Edinburgh EHI $1 H N$, Scotland, UK
}

Received December 1991

Revised July 1992

Abstract

Bundy, A., A. Stevens, F. van Harmelen, A. Ireland and A. Smaill, Rippling: a heuristic for guiding inductive proofs, Artificial Intelligence 62 (1993) 185-253.

We describe rippling: a tactic for the heuristic control of the key part of proofs by mathematical induction. This tactic significantly reduces the search for a proof of a wide variety of inductive theorems. We first present a basic version of rippling, followed by various extensions which are necessary to capture larger classes of inductive proofs. Finally, we present a generalised form of rippling which embodies these extensions as special cases. We prove that generalised rippling always terminates, and we discuss the implementation of the tactic and its relation with other inductive proof search heuristics.

\section{Introduction}

\subsection{Motivation}

One of the major problems facing artificial intelligence research is how to control search to avoid the combinatorial explosion. This paper describes rippling, a very successful heuristic for controlling search in inductive proof, a domain in which the combinatorial explosion takes an extreme form:

Correspondence to: A. Bundy, Department of Artificial Intelligence, University of Edinburgh, 80 South Bridge, Edinburgh EH1 1HN, Scotland, UK. Telephone: $(+44-31) 650$ 2716. Fax: (+44-31)6506516. E-mail: bundy@ed.ac.uk.

*Current affiliation: Oxford University Computing Laboratory, Oxford, UK.

** Current affiliation: Department of Social Science Informatics (SWI), University of Amsterdam, Amsterdam, Netherlands. 


$$
\begin{aligned}
& \frac{P(0), \forall n: \text { nat. }(P(n) \rightarrow P(s(n)))}{\forall n: n a t . P(n)} \\
& \frac{\forall x: \tau .(\forall y: \tau . y \prec x \rightarrow P(y)) \rightarrow P(x)}{\forall x: \tau . P(x)}
\end{aligned}
$$

Fig. 1. Rules of mathematical induction. Two example rules of mathematical induction are shown. The first is the simplest induction rule: Peano induction for natural numbers. The second is a general schema for well-founded induction. The relation $\prec$ is a well-founded order on the type $\tau$. We can formalise induction either as an unlimited number of variations on the first rule or by using the second rule as a schema in which $\tau$ and $\prec$ can be instantiated in an infinite number of ways. The expression $x: \tau$ is to be read as $x$ is of type $\tau$.

its search spaces have infinite branch points. Inductive proofs employ one or more of the infinitely many forms of mathematical induction ${ }^{1}$ (see Fig. 1). Moreover, inductive proofs sometimes require the generalisation of the current goal formula to any formula that implies it. Both of these proof steps involve infinite branching.

Rippling was designed as a domain-specific heuristic. It controls a particular part of inductive proofs-a part that does not even involve infinite branching. It might, therefore, be considered to be of limited interest. We hope to show otherwise.

- Inductive proof is a much more important inference technique than its relative neglect would imply. It is required whenever it is necessary to reason about repetition. This may occur in mathematics, programming, electronics, planning, etc. Thus induction is used in plan formation and in hardware and software verification, synthesis, and transformation. There are unexplored applications in explanation-based generalisation and nonmonotonic reasoning.

- Although rippling controls only part of an inductive proof many of the other control problems that arise can be made by asking "how can I make this choice so that rippling will be facilitated?". In this way, rippling can help choose an appropriate induction rule, find an appropriate generalisation, conjecture a missing lemma, and instantiate an existential witness. We are developing a technique called middleout reasoning to formalise this indirect mode of search control (see Section 13.4).

- Rippling is proving to be applicable to reasoning problems outside inductive proof. It can be used whenever the current goal is structurally similar to some previous theorem, assumption, or axiom. See

\footnotetext{
${ }^{1}$ Not to be confused with the learning form of induction, which is a rule for conjecturing general conclusions from particular examples.
} 
Section 13.6 for an application of rippling to summing series.

- Last but not least, this study of rippling is an exemplar of a new approach to heuristic search. We are not content to report the empirical success of a rule of thumb, but ask "why does it work?". The deeper understanding we gain by answering this question informs our exploration for improved heuristics. It also sheds light on the skills that may be involved in successful reasoning. Moreover, this understanding can itself be implemented in our reasoning program as a process of metalevel reasoning. This may lead to a program that can analyse its own failure and adjust its behaviour accordingly.

\subsection{Background}

In [4] we introduced the concept of proof plans and gave a simple example proof plan for guiding inductive proofs. A proof plan is an outline of a family of proofs which can be used to guide the search for proofs in this family. Proof planning is a technique for the global control of proof search. Many simple inductive proofs fit the outline of our inductive proof plan. Figure 2 describes its basic structure.

The key idea of this inductive proof plan is a tactic for manipulating the induction conclusion to enable the induction hypothesis to be used in its proof. Following Aubin [1] we initially called this tactic rippleout. In [6] we described an implementation of this inductive proof plan within Oyster-CLAM, a theorem prover for a higher-order, intuitionist, typed logic, and we reported its performance on various standard example inductive theorems. Oyster is a reimplementation in Prolog of the Nuprl interactive proof editor, [12]. Our tactics are Prolog programs which drive Oyster by applying its rules of inference. A proof plan is a tactic together with its method. A method partially specifies a tactic. It consists of the preconditions under which the tactic is applicable and the effects of its application. These are written in a meta-logic. CLAM is a plan formation program which uses these methods to build a specialpurpose proof plan for each theorem from a set of general-purpose proof plans.

In [7] we described the relationship between our inductive proof plan and the technique used by Boyer and Moore [3] to choose an appropriate induction rule and induction variable. We showed how we rationally reconstructed the Boyer-Moore technique as a process of look-ahead whose purpose is to maximise the chances that rippling-out would succeed.

We have tested our implementation extensively on a large number of inductive theorems, most of which are drawn from the Boyer-Moore corpus [3, Appendix A] adapting the original proof plan as necessary. This paper reports the result of that study. The spirit of the proof plan has survived 
these tests, with rippling-out remaining the central idea of the proof plan. However, the tests have suggested principled extensions to the proof plan, which preserve the essential intuition behind it while increasing its range of application. The main result is a generalisation of the ripple-out tactic. Since this generalisation includes rippling in other directions than "out", we have named the generalised tactic, ripple.

\subsection{Outline}

In this paper we give a complete account of rippling which supersedes earlier accounts in $[6,9]$. We start with a simple introduction to the original ripple-out tactic (Section 2) and then motivate and explain various extensions (Sections 3-9). These include the generalised ripple tactic, which embodies all but one of these extensions in a uniform framework (Section 8). We describe the implementation of the ripple tactic in the Oyster-CLAM system (Section 10) and give some experimental results of this implementation (Section 11). We compare rippling to rival techniques (Section 12), suggest some avenues for further research (Section 13) and draw some conclusions (Section 14). We show that ripple terminates (Appendix A).

The extensions to rippling-out reported below are: rippling with multiwave-rules (Section 3), rippling-in (Section 4), rippling with conditional wave-rules (Section 5), rippling-sideways (Section 6), rippling-across (Section 7) and rippling under existential quantifiers (Section 9). Each extension is illustrated with simple examples of proofs which require them. We argue that the extensions are natural improvements of the original idea.

\section{The basic rippling-out tactic}

In this section we briefly introduce the main idea of rippling-out and the role it plays within our inductive proof plan (see Fig. 2).

To understand rippling-out imagine a loch ${ }^{2}$ in which the induction conclusion appears as a reflection of the hypothesis. The reflection is not a perfect image of the original because wherever the induction variable appears in the induction hypothesis, the induction term appears in the induction conclusion. The expressions which appear in the induction conclusion, but not in the induction hypothesis, we call wave-fronts. The rest of the induction conclusion, i.e. the expressions which do appear also in the induction hypothesis, we call the skeleton, following [15]. Wave-fronts are like ripples

\footnotetext{
${ }^{2}$ The Scottish word for "lake".
} 


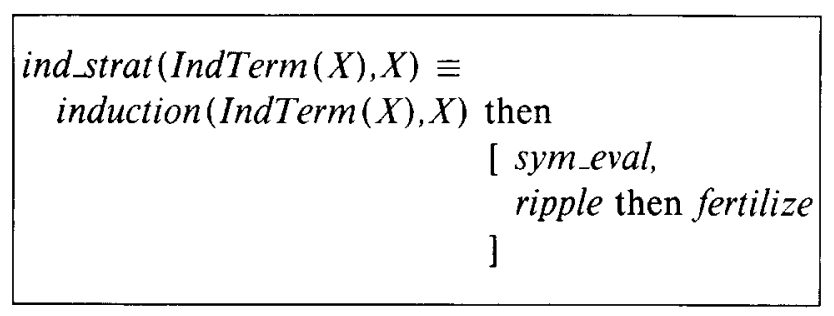

Fig. 2. The inductive proof plan. $X$ is the induction variable and IndTerm is the wave-front introduced into the induction conclusion by the induction rule of inference. Induction rules are indexed by their IndTerms. The application of the induction tactic exchanges the original theorem for a list of base and step cases: only one of each is shown in the figure. The sym_eval tactic is applied to the base case. This tactic is a combination of symbolic evaluation, tautology checking, equality substitution, etc. The ripple tactic is applied first to the step case. This creates a copy of the induction hypothesis within the induction conclusion. The fertilize tactic then uses the induction hypothesis to prove the induction conclusion. Any remaining subgoals are proved recursively using sym_eval and ind_strat.

on the surface of the loch, which spoil the reflection. Consider, for instance, a simple proof of the associativity of,+ 3

$$
\forall X: \text { nat. } \forall Y: \text { nat. } \forall Z: \text { nat. } X+(Y+Z)=(X+Y)+Z,
$$

by successor induction on $X$. The induction hypothesis is

$$
x+(y+z)=(x+y)+z
$$

where $x, y$, and $z$ represent skolem constants. ${ }^{4}$ The induction conclusion is

$$
s(\underline{x})+(y+z)=(s(\underline{x})+y)+z .
$$

The induction term is $s(x)$ and the $s(\cdots)$ constructor function is the wavefront. $x+(y+z)=(x+y)+z$ is the skeleton.

\subsection{Wave-fronts and wave-rules}

More generally, a wave-front is a term from which a proper subterm is deleted, i.e. a sequence of nested functions with the innermost argument removed. This innermost argument (the $x$ in (2)) is the wave-hole. When the wave-hole is filled by a term, the wave-front is said to dominate the term. We adopt the convention that wave-fronts are indicated by boxes with the wave-holes underlined, as in the example above. An alternative graphical representation of wave-fronts is given in Fig. 3.

\footnotetext{
${ }^{3}$ To improve readability we have translated the type-theoretic logic used by Oyster-CIAM into a more conventional notation.

${ }^{4}$ We adopt the Prolog convention that identifiers starting with upper case letters indicate variables and those starting with lower case letters indicate constants.
} 
Initially, the wave-fronts are functions which immediately dominate the induction variable. The role of rippling-out is to move them outwards-just like the ripples on a loch-leaving behind them an unspoiled reflection of the induction hypothesis. Rippling-out works by backwards reasoning from the induction conclusion to the induction hypothesis using wave-rules. A wave-rule is a rewrite rule of the form,

$$
\eta(\underline{\xi(\underline{\mu}))}) \Rightarrow \zeta(\underline{\eta(\mu))},
$$

where $\eta, \xi$, and $\zeta$ are terms ${ }^{5}$ with one distinguished argument. $\mu$ is usually a variable, but can be any term. $\zeta$ may be empty, but $\xi$ and $\eta$ must not be. $\xi$ and $\zeta$ are called the old and new wave-fronts, respectively. Note that the effect of applying such a rule is to move the old wave-front $\xi$, in the induction conclusion, outwards past the $\eta$ and to turn it into a new wave-front $\zeta$.

We adopt the convention that $\Rightarrow$ stands for rewriting and $\rightarrow$ stands for implication. Recall that rippling-out reasons backwards: from the theorem towards the axioms. To apply the implication $\phi \rightarrow \psi$ it uses the rewrite rule $\psi \Rightarrow \phi$. Thus the left/right orientation of implication is opposite to that of rewriting.

This general form includes all rewrite rules formed from the step cases of recursive definitions. Restricted to such recursive rules, rippling-out is a constrained version of unfolding or symbolic evaluation. But, as we will see, wave-rules can also be formed from non-recursive definitions and from lemmas, so rippling-out extends unfolding. CLAM analyses all the definitions given to, and lemmas proved by, Oyster and transforms them into wave-rules in as many ways as possible. Examples of wave-rules are given below.

Example 1 (Wave-rules).

$$
\begin{aligned}
& s(\underline{U})+V \Rightarrow s(\underline{U+V}) \\
& s(\underline{U}) \times V \Rightarrow \underline{U \times V+V} \\
& \operatorname{even}(s(s(\underline{U})) \Rightarrow \operatorname{even}(U) \\
& U+(\underline{V}+W) \Rightarrow(\underline{U}+V)+W \\
& (U+\underline{V})+W \Rightarrow U+(\underline{V}+W)
\end{aligned}
$$

Wave-rules (4), (5) and (6) are formed from recursive definitions. Waverules (7) and (8) are both formed from the associative law of + , which is

\footnotetext{
${ }^{5}$ We adopt the convention of using Greek letters for meta-symbols, e.g. for describing the patterns of wave-rules.
} 
not a recursive definition. Note that the wave-front of rule (6) is compound, i.e. contains more than one function symbol, and that the $\zeta$ part of this rule is empty. Note also that rules (7) and (8) show that an equation can give rise to several different wave-rules and that these can be oriented in different directions provided that the wave-front annotations are different.

Definition 1 (Preconditions of applying wave-rules). The preconditions of applying a wave-rule to rewrite a subexpression of the induction conclusion are that:

- the left-hand side of the rule matches the subexpression;

- the subexpression contains at least one wave-front; and

- this wave-front in the subexpression is matched with the old wave-front in the rule.

These preconditions ensure that wave-rules always move wave-fronts outwards towards the root of the skeleton. Thus wave-rules are applied selectively, not exhaustively.

Repeated application of rule (4) to induction conclusion (2) ripples the two wave-fronts to right outside the left- and the right-hand terms of the induction conclusion, as follows:

$$
\begin{aligned}
& s(\underline{x})+(y+z)=(s(\underline{x})+y)+z, \\
& s(\underline{x+(y+z)})=s(\underline{x+y})+z, \\
& s(\underline{x+(y+z)})=s(\underline{(x+y)+z) .} .
\end{aligned}
$$

A graphical representation of the rippling-out of the right-hand side is given in Fig. 3.

\subsection{Kinds of termination}

The basic version of rippling-out outlined in this section applies only to induction conclusions that are equations or equivalencies. Wave-fronts are rippled out until they dominate the left- and right-hand sides of these induction conclusions.

The movement of wave-fronts can terminate in three ways.

- If a wave-front is moved to dominate the left- or right-hand term of the induction conclusion, then we say it is beached. No further rippling-out of this wave-front is, then possible or necessary. Two examples can be found in equation (9), above.

- If a wave-rule is applied in which $\zeta$ is empty, then there is no new wavefront. We say it peters out. Two examples can be found in equation (21), Section 3, below. 


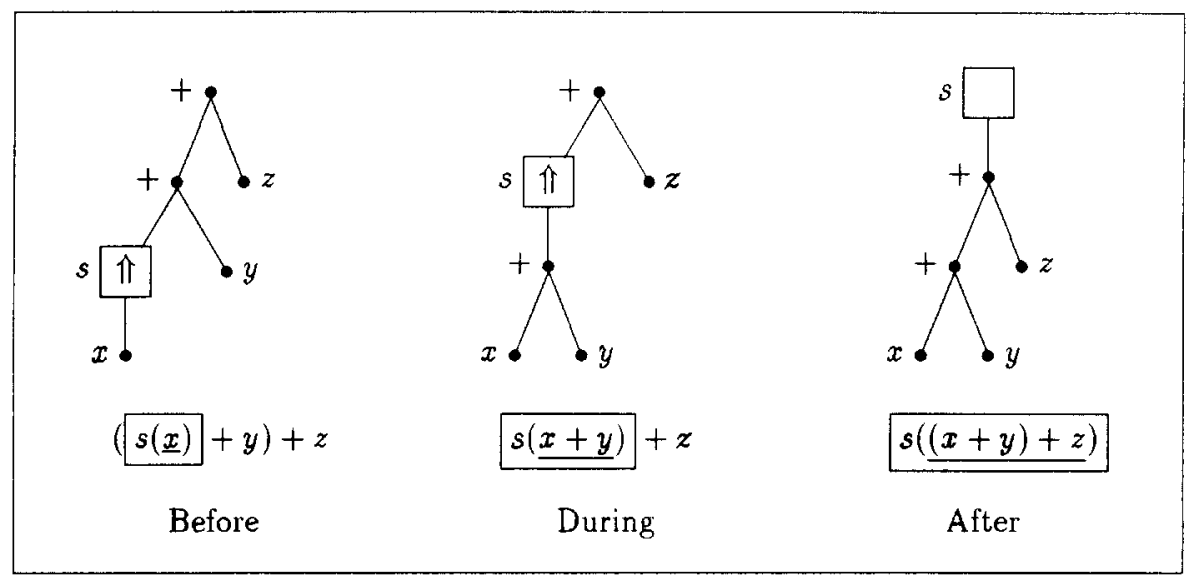

Fig. 3. Rippling out $(s(x)+y)+z$. The three trees show the term before, during and after rippling-out. Each tree represents the term as an expression tree. The nodes of each tree are labelled by a function or predicate symbol or a constant or variable. A function or predicate of arity $n$ has $n$ descendent subtrees: one for each of its arguments. Wave-fronts are indicated by square nodes and all other nodes by dots. Note that the three trees differ from each other only in the position of the square nodes. These square nodes are higher in each successive tree; the "After" one being beached at the top. The $\uparrow$ 's in the square nodes indicate the direction of rippling-out.

- If a wave-front is not beached, but no wave-rule applies to it, then we say the wave-front is blocked. An example can be found in equation (25), Section 4, below.

If all wave-fronts in a term are beached or petered out, then we say it is fully rippled. If all the wave-fronts are blocked, then we say that rippling-out is blocked.

When no further rippling-out is possible, then the induction hypothesis can often be used as a rewrite rule to produce an equation between two identical terms. Following Boyer and Moore, we call this tactic fertilize. The induction hypothesis may be used either way round. In our example we can use it left to right on the left-hand side of the induction conclusion, (9), to produce the equation:

$$
s(\underline{(x+y)+z)}=s(\underline{(x+y)+z)},
$$

which is readily proved, so completing the step case of the inductive proof.

\subsection{Search control}

Both rippling-out and fertilization are subject to careful control by our overall inductive proof plan.

- Rippling-out is applied to the induction conclusion immediately after the application of induction. The precondition of rippling-out ensures 
that it is applied only to move wave-fronts outwards. This greatly restricts the search space of rippling-out. It also ensures the termination of rippling-out, since the wave-fronts can only be moved outwards, through an unchanging skeleton, a finite number of times. This remains true even if the same equation is used as a wave-rule in different left/right orientations, cf. rules (7) and (8).

- Fertilization is applied to the induction conclusion when no further rippling-out is possible. It uses the induction hypothesis as a rewrite rule either left to right to the left-hand side of the induction conclusion or right to left to its right-hand side. If the wave-fronts on both sides of the induction conclusion are fully rippled, then either one of these is sufficient. If only one side is fully rippled, then this side is rewritten. If neither side is fully rippled, then fertilization is not possible.

\subsection{Merging and splitting}

Note that the general wave-rule format allows for wave-fronts to be compound terms. This opens up the possibility that compound wave-fronts might be split into several sub-wave-fronts and each of these rippled out separately. For instance, suppose the following compound wave-front appears in the induction conclusion

$$
\cdots \operatorname{even}(s(s(\underline{x}))+y) \cdots .
$$

As it stands this wave-front is blocked as none of the wave-rules we have considered so far meets the preconditions of rippling-out. However, if we split the wave-front into two,

$$
\cdots \operatorname{even}(s(\underline{\underline{s(\underline{x})})})+y) \cdots
$$

then wave-rule (4) will apply twice to produce first

$$
\cdots \operatorname{even}(s(\underline{\underline{s(\underline{x})]+y})}) \cdots
$$

and then

$$
\cdots \operatorname{even}(s(\underline{\underline{s(\underline{x+y})})}) \cdots
$$

Now once again rippling-out is blocked because no wave-rule meets its preconditions. This time the two adjacent wave-fronts must be merged into one,

$$
\cdots \operatorname{even}(s(s(\underline{x+y)})) \cdots
$$


so that wave-rule (6) will apply and produce

$$
\text { - even }(x+y) \cdots
$$

Splitting a compound wave-front and rippling each sub-wave-front separately can result in nested non-adjacent wave-fronts, e.g.

$$
\cdots \text { ᄀeven }(s(\underline{x})+y) \cdots .
$$

Note that the inner wave-front must always be in the wave-hole of the outer one. We say that the inner wave-front is beneath the outer one.

In CLAM this splitting and merging of wave-fronts is done on demand. First rippling-out is attempted with the current wave-front annotation. When this has been completed (either successfully or unsuccessfully) all possible splittings and mergings are made and rippling-out is tried with each of these in turn.

An alternative mechanism would be to hold all wave-fronts in a normal form, e.g. maximally split or maximally merged. The maximally split normal form is particularly attractive as it allows us to continue to use regular matching to ensure wave-front agreement. If the maximally merged normal form is used, then the matcher must be modified to dynamically split wavefronts as required. The INKA system records wave-fronts by annotating each symbol as either in the wave-front or in the skeleton [15]. This solution has some similarity to the maximally split normal form.

\subsection{Branching rates}

In practice, the branching rate of rippling-out is very low. The rippling-out of one wave-front is independent of the rippling-out of the others, so that they can be dealt with in a fixed order and redundant branching can be avoided. ${ }^{6}$ Genuine branching happens only when more than one wave-rule applies to the same wave-front. But this is rare.

To see why this is so consider how it might happen. Suppose the wavefront occurs in the context $\cdots \eta(\underline{\xi(\underline{\alpha}))}) \cdots$. In the simplest case there might be two wave-rules which both ripple $\xi$ out through $\eta$, i.e.

$$
\begin{aligned}
& \eta(\xi(\underline{\mu})) \Rightarrow \zeta_{1}(\eta(\mu)) \\
& \eta(\xi(\underline{\mu})) \Rightarrow \zeta_{2}(\eta(\mu)) .
\end{aligned}
$$

${ }^{6} \mathrm{This}$ is not implemented in the current version of CLAM. 
Suppose, without loss of generality, that these rules are both based on equations. Since the left-hand sides of the rules are equal, the right-hand sides are also equal, i.e.

$$
\zeta_{1}(\eta(\mu))=\zeta_{2}(\eta(\mu)) \text {. }
$$

So $\zeta_{1}$ and $\zeta_{2}$ are equal over the range of the function $\eta$. If we keep wavefronts on the right-hand sides of wave-rules in a simple normal form, then in most cases $\zeta_{1}$ and $\zeta_{2}$ will be identical. Thus it is rare to have distinct wave-rules with identical left-hand sides, as required for this simple form of branching.

The general wave-rule format allows for both $\eta$ and $\xi$ to be compound. In these cases branching is possible. However, the alternative branches will often lead to similar proofs. Consider, for instance, the situation where $\xi(\mu)$ has the compound form $\xi^{\prime}\left(\xi^{\prime \prime}(\mu)\right)$ and we have the alternative wave-rules:

$$
\begin{aligned}
& \eta\left(\xi^{\prime}\left(\xi^{\prime \prime}(\underline{\mu})\right)\right) \Rightarrow \zeta_{1}(\underline{\eta(\mu))}, \\
& \eta\left(\underline{\xi^{\prime}(\underline{\nu})}\right) \Rightarrow \zeta_{2}(\underline{\eta(\nu))},
\end{aligned}
$$

leading to two branches in the rippling-out search space. Note that if the second branch is not to immediately become blocked, there must also be a wave-rule of the form

$$
\eta\left(\underline{\left.\xi^{\prime \prime}(\underline{\mu})\right)} \Rightarrow \zeta_{3}(\underline{\eta(\mu))} .\right.
$$

So, by a similar calculation as in the simple case:

$$
\zeta_{1}(\eta(\mu))=\zeta_{2}\left(\zeta_{3}(\eta(\mu))\right),
$$

so that the alternative routes will lead to similar proofs. In this case it is not usually possible to remove the duplication of wave-rules by normalising the right-hand side wave-fronts.

As an example of this kind of branching consider the pair of wave-rules:

$$
\begin{aligned}
& \operatorname{even}(s(s(\underline{U}))) \Rightarrow \operatorname{even}(U), \\
& \operatorname{even}(s(\underline{U})) \Rightarrow \neg \operatorname{even}(U),
\end{aligned}
$$

where $\zeta_{1}$ is empty and both $\zeta_{2}$ and $\zeta_{3}$ are $\neg$. Note that neither $\zeta_{2}$ nor $\zeta_{3}$ can be normalised on their own, but in combination they normalise to $\zeta_{1}$, as expected.

The extensions to ripple-out described below will introduce new possibilities for branching. Nevertheless, this will remain a relatively rare phenomenon, so that, in practice, the search space of rippling is very small. 


\section{Multi-wave-rules and strong fertilization}

Our first extension to rippling-out is to allow wave-rules which ripple out more than one wave-front at once. We call these multi-wave-rules. They are highly desirable if one side of the induction conclusion contains more than one wave-front-as it will do if it contains more than one occurrence of the induction variable. They allow rippling to transform several wavefronts into one and, hence, continue rippling beyond the point at which the old version of rippling-out would have become blocked. They also allow us to strengthen rippling-out and fertilization and, thereby, apply them to induction conclusions that are not equations or equivalencies.

\subsection{General wave-rule format}

While we are making this extension we will also extend wave-rules to cope with wave-fronts containing more than one wave-hole and to the introduction of multiple skeletons. This leads to the generalisation of wave-rule format (3) to the following,

$$
\begin{aligned}
& \eta\left(\underline{\xi_{1}\left(\underline{\mu_{1}^{1}}, \ldots, \mu_{1}^{p_{1}}\right)}, \ldots, \xi_{n}\left(\underline{\left.\mu_{n}^{1}, \ldots, \mu_{n}^{p_{n}}\right)}\right)\right. \\
& \quad \Rightarrow \zeta\left(\eta\left(\varpi_{1}^{1}, \ldots, \varpi_{n}^{1}\right), \ldots, \eta\left(\varpi_{1}^{k}, \ldots, \varpi_{n}^{k}\right)\right)
\end{aligned}
$$

where each $\varpi_{i}^{j}$ is either an unrippled wave-front,

$$
\xi_{i}\left(\mu_{i}^{1}, \ldots, \mu_{i}^{p_{i}}\right)
$$

or is one of the wave-holes, $\mu_{i}^{l}$. For each $j$, at least one $\varpi_{i}^{j}$ must be a wavehole. As before, $\eta$, the $\xi_{i}$ 's and $\zeta$ are terms with distinguished arguments. $\zeta$ may be empty, but the $\xi_{i}$ 's and $\eta$ must not be. The $\xi_{i}$ 's are the old wave-fronts and $\zeta$ is the new wave-front. Note that the application of a multi-wave-rule with $k \eta$ 's in the right-hand side will replace one skeleton with $k$ skeletons in the induction conclusion. Some example multi-wave-rules are given below.

Example 2 (Multi-wave-rules).

$$
\begin{aligned}
& s(\underline{U})=s(V) \Rightarrow U=V \\
& s(\underline{U}) \geqslant s(\underline{V}) \Rightarrow U \geqslant V
\end{aligned}
$$

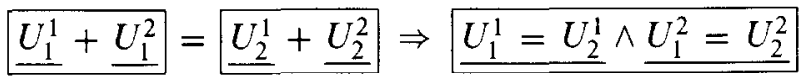

$$
\begin{aligned}
& \max \left(\underline{U_{1}^{1}}, \underline{U_{1}^{2}}\right) \geqslant \min \left(\underline{U_{2}^{1}}, \underline{U_{2}^{2}}\right) \Rightarrow \underline{U_{1}^{1} \geqslant U_{2}^{1}} \wedge \underline{U_{1}^{2} \geqslant U_{2}^{2}}
\end{aligned}
$$




$$
\begin{aligned}
& \underline{(\underline{U}+\underline{V})} \times W \Rightarrow \underline{U} \times W+\underline{V} \times W \\
& \max \_h t(\operatorname{tree}(\underline{U}, \underline{V})) \Rightarrow s\left(\max \left(\max h t(U), \max \_h t(V)\right)\right)
\end{aligned}
$$

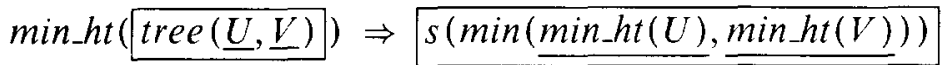

$$
\begin{aligned}
& \operatorname{binom}(s(\underline{U}), s(\underline{V})) \Rightarrow \operatorname{binom}(U, s(\underline{V}))+\operatorname{binom}(U, V)
\end{aligned}
$$

Note the occurrence of multiple $\mu$ 's, multiple $\eta$ 's and even multiple $\xi$ 's in these rules. The first five rules are further examples of wave-rules not formed from recursive definitions. Note that rules (15) and (16) are sound because the orientation of implication is right to left. Note also that in rule $(20)$ the wave-fronts are only partially removed from one of the new skeletons.

The advantages of multi-wave-rules of format (12) compared to simple wave-rules of format (3) are as follows.

- The generalisation of the single $\xi$ in (3) to the multiple $\xi_{i}$ 's in (12) enables the simultaneous rippling-out of multiple wave-fronts. However, some of these multiple $\xi_{i}$ 's may not be rippled out by some wave-rulescf. rule (20).

- The generalisation of the single $\mu$ to the multiple $\mu_{i}$ 's enables the rippling-out of wave-fronts containing more than one wave-hole. However, we may sometimes want to treat some of these holes as part of the wave-front-see Section 3.4 below.

- The generalisation of the single $\eta$ argument of $\zeta$ to the multiple $\eta$ arguments enables several different induction hypotheses to fertilize the induction conclusion.

These last two extensions go together; multiple $\mu$ 's tend to create multiple $\eta$ 's, each one making a different selection of $\mu$ 's for its arguments.

Following this generalisation of the wave-rule format it is necessary to revise the last item of the preconditions of wave-rule application (Definition 1). This is the first of a series of revisions which will be required as the concept of rippling evolves throughout this paper. We will adopt the convention of highlighting the changes by using italic font.

Definition 2 (Preconditions of applying multi-wave-rules). The preconditions of applying a multi-wave-rule to rewrite a subexpression of the induction conclusion are that:

- the left-hand side of the rule matches the subexpression;

- the subexpression contains at least one wave-front; and 
- each wave-front in the subexpression is matched with an old wave-front in the rule.

\subsection{Strong fertilization}

We can now revisit and reconsider the associativity of + example from Section 2. Instead of applying fertilization to the induction conclusion (9), we can use the multi-wave-rule (13) to continue the rippling-out and infer

$$
x+(y+z)=(x+y)+z,
$$

which is identical to the induction hypothesis, (1), i.e. the wave-fronts have petered out. The proof thus terminates by direct appeal to the induction hypothesis. This direct use of the induction hypothesis constitutes a new form of fertilization which we will call strong fertilization. The form of fertilization described above in Section 2 we will rename weak fertilization. Since strong fertilization applies to induction conclusions of any form (and not just to equalities and equivalencies) it is more generally applicable, and its application will normally be the purpose of rippling-out. However, weak fertilization must still be retained to cope with equational or equivalential induction conclusions that get blocked on one side.

\subsection{A worked example}

To illustrate the use of multi-wave-rules to prove non-equational theorems with the aid of multiple induction variables and multiple induction hypotheses, consider the following example.

$$
\forall T: \text { tree. } \max _{-} h t(T) \geqslant \min h t(T),
$$

where $\max h t(T)$ is the length of the longest path in a binary tree, $T$, and $\min \_h t(T)$ is the length of the shortest. Trees will be built from the constructor functions leaf $(T)$ and tree $\left(T_{1}, T_{2}\right)$. This example has been designed to exhibit all the features of multi-wave rippling, and as a consequence is rather artificial.

Our proof will use the standard, structural induction rule on binary trees, i.e. the theorem is proved for the empty tree and then assumed for the left and right subtrees and proved for the whole tree. Thus, in the step case we will have the following two induction hypotheses

$$
\begin{aligned}
& \max \_h t(l) \geqslant \min _{-} h t(l), \\
& \max _{-} h t(r) \geqslant \min _{-} h t(r),
\end{aligned}
$$

and must prove the induction conclusion,

$$
\max \_h t\left([\operatorname{tree}(\underline{l}, \underline{r})) \geqslant \min \_h t(\operatorname{tree}(\underline{l}, \underline{r})]\right) \text {, }
$$


in which both $l$ and $r$ are induction variables.

To ripple this induction conclusion out we will use the multi-wave-rules from Example 2,

$$
\begin{aligned}
& s\left(\max \left(\max \_h t(l), \max \_h t(r)\right)\right) \\
& \geqslant s(\min (\min h t(l), \min h t(r))) \text {, } \\
& \max (\text { max_ht }(l), \text { max_ht }(r)) \\
& \geqslant \min (\text { min_ht }(l), \text { min_ht }(r)) \text {, }
\end{aligned}
$$

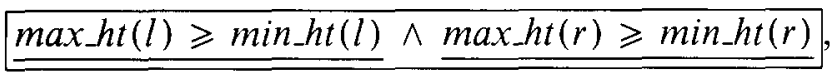

to which strong fertilization applies, finishing the proof of the step case.

Note the following points from this proof.

- Because the constructor function tree contains two induction variables, $l$ and $r$, it forms a wave-front with two wave-holes. This situation is inherited by subsequent versions of the induction conclusion, with $\max$, $\min$, and $\wedge$ also having two-hole wave-fronts.

- The theorem is not an equation or equivalence, so strong fertilization must be used to complete the rippling-out.

- The skeleton is duplicated by the first wave-rule. This eventually leads to two copies of the induction hypothesis, which are fertilized independently.

- The two wave-fronts in line (23) are compound terms. They are each split into two wave-fronts and rippled out separately.

- The last wave-front is a logical connective. Rippling-out can proceed through predicates and connectives as well as functions.

\subsection{Rule weakening}

It is sometimes desirable to use multi-wave-rules at less than their full strength, i.e. to treat some of the wave-holes as part of the wave-front. We can see two examples of this in the following proof of the associativity of $x$,

$$
\forall X: \text { nat. } \forall Y: \text { nat. } \forall Z: \text { nat. } X \times(Y \times Z)=(X \times Y) \times Z \text {. }
$$

The rippling of the step case uses rule (5) from Example 1 and rules (15) and (17) from Example 2. 


$$
\begin{aligned}
& s(\underline{x}) \times(y \times z)=(\underline{s(\underline{x}) \times y) \times z,} \\
& \underline{x \times(y \times z)}+y \times z=((\underline{\underline{x} \times y})+y) \times z, \\
& x \times(y \times z)+y \times z=(\underline{x \times y) \times z}+y \times z, \\
& x \times(y \times z)=(x \times y) \times z \wedge y \times z=y \times z .
\end{aligned}
$$

Note that wave-rules (15) and (17) are used as if their wave-fronts were

$$
\begin{aligned}
& \underline{\underline{U_{1}^{1}}+U_{1}^{2}}=\underline{U_{2}^{1}+U_{2}^{2} \Rightarrow \underline{U_{1}^{1}=U_{2}^{1}} \wedge U_{1}^{2}=U_{2}^{2},} \\
& (\underline{U}+V) \times W \Rightarrow \underline{U \times W}+V \times W,
\end{aligned}
$$

i.e. with the second wave-hole merged with the wave-front on both leftand right-hand sides of each rule. This is called wave-rule weakening. We currently implement weakening at compile time, i.e. when it is storing a new wave-rule CIAM also calculates all its possible weakenings and stores these as well. We are also exploring the implementation of weakening by modification of the matcher, i.e. by allowing it to ignore wave-fronts in the wave-rule.

The weakening of wave-rules can result in the capture of an inner wavefront nested beneath an outer one. The inner one goes from being in the wave-hole of the outer one to being within its wave-front proper. We say the inner wave-front is inside the outer one. Contrast this situation with the nesting beneath described in Section 2.4. Inside wave-fronts are merged with the outer wave-front-all their wave-front annotation being dropped.

\subsection{Simplification of wave-fronts}

Some of the multi-wave-rules call for identical wave-fronts at different positions with the induction conclusion, e.g. rule (13) in Example 2 calls for each side of an equation to start with $s(\underline{\ldots})$. As a result of earlier rewriting it sometimes happens that an induction conclusion has equal, but not identical, wave-fronts at these positions. An example occurs during the proof of the distributivity law of $\times$ over + ,

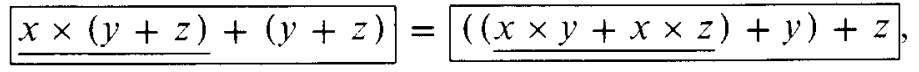

which prevents the application of rule (15).

To reduce the chances of this problem blocking a ripple, CIAM regularly simplifies wave-fronts by applying a simple normalisation tactic to them. The details of this normalisation are beyond the scope of this paper, but it consists of symbolic evaluation with recursive definitions and other complexityreducing rewrite rules. Note that this normalisation process must be applied to the wave-fronts without perturbing their wave-holes. These wave-holes 
form part of the skeleton and any perturbation will prevent the application of strong fertilization, unless it is applied uniformly to all skeletons and to the induction hypothesis.

\section{Weak fertilization and rippling-in}

\subsection{Example of a blocked ripple}

Weak fertilization is useful when a rippling process gets blocked on one side of an equation or equivalence and strong fertilization is not possible. Consider, for instance, the theorem

$$
\forall X: \text { nat. half }(X+X)=X,
$$

where half is the half integer function defined by:

$$
\begin{aligned}
& \operatorname{half}(0)=0, \\
& \operatorname{half}(s(0))=0, \\
& \operatorname{half}(s(s(U)))=s(\operatorname{half}(U))
\end{aligned}
$$

This definition provides the wave-rule:

$$
\text { half }(s(s(\underline{U}))) \Rightarrow s(\text { half }(U)) .
$$

Suppose we try to prove the theorem with $s(x)$ induction, so the induction hypothesis is

$$
\operatorname{half}(x+x)=x
$$

and the induction conclusion is

$$
\text { half }(s(\underline{x})+s(\underline{x}))=s(\underline{x}) .
$$

The rule (4) applies to the left-hand side of the induction conclusion, which ripples the left-most wave-front once. The induction conclusion becomes

$$
\operatorname{half}(s(x+s(\underline{x}))]=s(\underline{x}) \text {. }
$$

At this point no further wave-rules apply and the ripple is blocked. Note that the wave-rule required to unblock this ripple is

$$
U+s(\underline{V}) \Rightarrow s(\underline{U+V})
$$

a commuted version of (4). For the sake of this example we are assuming that this rule is not available.

Fortunately, the right-hand side of $(25)$ is trivially fully rippled, so we can apply weak fertilization to get 


$$
\text { half }(s(x+s(x)))=s(\text { half }(x+x)) .
$$

After strong fertilization, wave-fronts are removed since they have fully completed their job. After weak fertilization they may still have a role to perform on the unblocked side of the equation, i.e. the right-hand side in this example, so we have left them in place on this side. Their residual role is to be rippled in by applying reversible wave-rules right to left. We have indicated this by annotating the wave-front with the direction in which it should be rippled: inwards. ${ }^{7}$ Wave-fronts that should be rippled outwards will be annotated by an upwards arrow. Rippling-in is our second extension to rippling-out.

\subsection{Why rippling-in is a good idea}

To see why rippling-in is a good idea, consider the following generic induction step case. The induction hypothesis has the following schematic form:

$$
++++++=* * * * * * *
$$

and the induction conclusion is:

$$
\left.+++\underline{?+?}{ }^{\uparrow}+++=* * * \underline{?}^{\uparrow}\right]^{\uparrow} * * * .
$$

Suppose that the right-hand side of the induction conclusion can be fully rippled but the left-hand side becomes blocked:

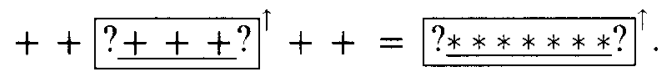

Fertilizing the right-hand side gives:

$$
++?++?++=?+++++++?
$$

Note that the +++++++ skeletons on either side of the $=$ are identical, but that the ?? wave-fronts may differ. The intuition is that since the two sides of equation (29) are so similar it will be easier to prove than the original equation, (27), in which the two sides may be completely different.

If the wave-front surrounding the right-hand side can be rippled in towards the induction variable, then the induction conclusion can be transformed to the form

$$
++?++?++=+?+++++?+,
$$

where the wave-front has been dropped since no further rippling-in is possible. At this point the outermost function symbols on the left and right are

${ }^{7}$ Or downwards in the graphical representation. 
bound to be identical since they each belong to identical +++++++ skeletons. Thus, they may be cancelled, reducing the induction conclusion to

$$
+?++?+=?+++++? \text {. }
$$

The aim of rippling-in is to enable this cancellation to take place. In CLAM, rippling-in and cancellation are interleaved until no further cancellation is possible. The intuition is that equation $(30)$ is even easier to prove than equation (29) because it is syntactically simpler, while retaining the property that the two sides of the equation are very similar.

Another way to look at rippling-in is as a technique for bi-directional rippling-out. Ideally, both sides of the equation would ripple-out fully. The left-hand side would then have the form,

$$
?+++++++?
$$

which is the same form as the right-hand side after fertilization, cf. equation (29). Unfortunately, the left-hand side gets blocked at:

$$
++\underline{?++?}^{\uparrow}++
$$

Rippling-in starts from

$$
?+++++++?
$$

and performs what would have been the last few rippling-outs in reverse to get:

$$
+\underline{+++++?}{ }^{1}+
$$

Note that the final subgoal, equation (30), could be made into a wave-rule. In fact, if this wave-rule were available the rippling-out would not have been blocked, since it is just what is needed to continue rippling the left-hand side of equation (28). It is the missing wave-rule-or, more accurately, an instance of one such missing wave-rule.

\subsection{Example of rippling-in and lemma discovery}

To illustrate rippling-in we return to our half integer example just after weak fertilization:

$$
\text { half }(s(x+s(x)))=s \underline{\text { half }(x+x))}
$$

Rippling-in can be performed by applying wave-rule (24) right to left. This gives

$$
\operatorname{half}(s(x+s(x)))=\operatorname{hal}\left(\underline{s(s(\underline{x+x}))}^{\downarrow}\right),
$$


after which further rippling-in would not assist cancellation. The wave-fronts are now dropped and the outermost function symbols cancelled to give

$$
x+s(x)=s(x+x) .
$$

This remaining subgoal is proved by generalising the second occurrence of $x$ on each side of the equation to $y$ and then using $s(x)$ induction. The details of this generalisation step are beyond the scope of this paper.

Note that the subgoal (31) that remains, after weak fertilization and rippling-in, is an instance of the missing wave-rule (26). This is not a coincidence. In fact, we can see from the above analysis that if a ripple is blocked for the lack of a single wave-rule, and if weak fertilization and rippling-in succeed, then the subgoal they leave is bound to be an instance of the missing rule. In proving this subgoal, CLAM often starts by generalising it into the missing wave-rule. After the proof it is added to CLAM's stock of wave-rules for future use, so ripples will not block for the lack of this rule in future. We will refer to this phenomenon as the 'in-line' proof of wave-rules. It shows the power of our proof plans technique to conjecture and prove lemmas needed to complete a proof.

\subsection{Controlling rippling-in}

Controlling rippling-in raises a couple of complications that are not already present in rippling-out.

- Firstly, there may be a choice between different inwards directions. For instance, an expression $\eta\left(\underline{\mu(\alpha, \beta))}{ }^{\downarrow}\right.$ may be rippled in to

$$
\text { either } \mu\left({\underline{\eta^{\prime}(\underline{\alpha})}}^{\downarrow}, \beta\right) \text { or } \mu\left(\alpha,{\underline{\eta^{\prime \prime}(\underline{\beta})}}^{\downarrow}\right) \text {. }
$$

Fortunately, we can be guided by the shape of the other side of the equation. For cancellation to be possible this should have either the form $\mu(\alpha, \ldots)$ or the form $\mu(\ldots, \beta)$, and whichever it is will determine which rippling-in we perform.

- Secondly, we need to know when to stop. Again, we only ripple in if this will allow cancellation to take place. For instance, if the equation is

$$
\mu\left(\alpha, \eta^{\prime \prime}(\beta)\right)=\eta(\underline{\mu(\alpha, \beta))}
$$

and the only available wave-rule will ripple in the right-hand side to

$$
\mu\left({\underline{\eta^{\prime}(\underline{\alpha})}}^{\downarrow}, \beta\right),
$$

then rippling-in is stopped. 
- Thirdly, not all wave-rules can be legally applied right to left, e.g. if they are formed from implications, then they can only be used left to right on subformulae of positive polarity and right to left on subformulae of negative polarity. Similar remarks hold for inequalities. We call such wave-rules irreversible. Note that wave-rules formed from equalities and equivalencies are always reversible. For irreversible wave-rules CIAM uses a general notion of polarity to determine in which orientation and to which subexpressions they can be legally applied. For instance, for wave-rules formed from implications the polarity of a subformulae is the parity of the number of implicit or explicit negations within which it is contained, e.g. in $\neg p \wedge q \rightarrow r, p$ and $r$ are of positive polarity and $q$ is of negative polarity. For wave-rules formed from inequalities the polarity corresponds to the monotonicity of the functions containing the subexpression.

In Sections 6.3 and 11.2.2 we will meet a variation of rippling-in that uses a different kind of wave-rule.

\section{Conditional wave rules}

Our third extension is to allow conditional wave-rules, i.e. wave-rules that are only true under some condition. They have the form:

$$
\text { Cond } \rightarrow \text { LHS } \Rightarrow \text { RHS, }
$$

where $L H S \Rightarrow R H S$ is a wave-rule and Cond is a formula.

If the condition of a rule is provable from the current hypotheses, then, clearly, we can use the rule. But even if it is not currently provable we can still use the rule provided we divide the proof into two cases using the condition and its negation. The condition is then trivially provable in the first case. So a major problem to be solved in the use of conditional rules is when to try to prove the condition within the current case (either immediately or later) and when to use the condition to split the current case into two subcases.

As a partial solution to this problem, related conditional rules are stored, by CLAM , in covering sets of the form

$$
\begin{aligned}
& \operatorname{Cond}_{1} \rightarrow \\
& \quad \eta\left(\xi_{1}^{1}\left(\underline{\mu_{1}^{1}}, \ldots, \underline{\mu_{1}^{p_{1}}}\right), \ldots, \xi_{n}^{1}\left(\underline{\mu_{n}^{1}}, \ldots, \underline{\mu_{n}^{p_{n}}}\right)\right) \Rightarrow R H S_{1}, \\
& \quad \vdots \\
& \operatorname{Cond}_{k} \rightarrow \\
& \quad \eta\left(\xi _ { 1 } ^ { k } \left(\underline{\left.\mu_{1}^{1}, \ldots, \underline{\mu_{1}^{p_{1}}}\right), \ldots, \xi_{n}^{k}\left(\underline{\mu_{n}^{1}}, \ldots, \underline{\mu_{n}^{p_{n}}}\right) \Rightarrow R H S_{k},}\right.\right.
\end{aligned}
$$


where $R H S_{i}$ is either of the form

$$
\zeta_{l}\left(\eta\left(\mu_{1}^{q_{1}^{i}}, \ldots, \mu_{n}^{q_{n}^{i}}\right), \ldots, \eta\left(\mu_{1}^{r_{1}^{i}}, \ldots, \mu_{n}^{r_{n}^{i}}\right)\right)
$$

with $1 \leqslant q_{j}^{i}, r_{j}^{i} \leqslant p_{j}$ for $1 \leqslant j \leqslant n$ and $1 \leqslant i \leqslant k$, or is an expression not containing $\eta$ and where we require that

$$
\text { Cond }_{1} \vee \cdots \vee \text { Cond }_{k}
$$

and where for each $1 \leqslant i \leqslant n$ there exists a wave-front $\xi_{i}$ which subsumes the wave-fronts $\xi_{i}^{j}$ for all $1 \leqslant j \leqslant k$, where the $\xi_{i}$ 's are the wave-fronts to be rippled out in the induction conclusion. A wave-front $\xi$ subsumes a wave-front $\xi^{\prime}$ if $\xi$ consists of nested copies of $\xi^{\prime}$. For instance, the wave-front $s(s(\cdots))$ subsumes the wave-front $s(\cdots)$. Note that two consecutive ripples of $s(\cdots)$ will ripple $s(s(\cdots))$ once.

Note that it is not always possible to form a covering set from conditional wave-rules alone. Some conditional rules in a covering set will be conditional wave-rules and some will be conditional, non-recursive definitions of $\eta$. See, for instance, the covering set for $\in$ in Example 3 .

Now that wave-rules can be conditional it is necessary to add an additional item to the preconditions of wave-rule application (Definition 2).

Definition 3 (Preconditions of applying conditional wave-rules). The preconditions of applying a conditional wave-rule to rewrite a subexpression of the induction conclusion are that:

- the left-hand side of the rule matches the subexpression;

- the subexpression contains at least one wave-front;

- each wave-front in the subexpression is matched with a wave-front in the rule; and

- the condition of the wave-rule is provable from the current hypotheses.

Some examples of conditional rules are given below.

Example 3 (Conditional wave-rules).

$$
\begin{aligned}
& E l=H \rightarrow E l \in(H:: \underline{T}) \Rightarrow \text { true, } \\
& E l \neq H \rightarrow E l \in(H:: \underline{T}) \Rightarrow E l \in T ; \\
& H \in S \rightarrow(H:: \underline{T}) \cap S \Rightarrow H:: \underline{(T \cap S}), \\
& \neg H \in S \rightarrow(H:: \underline{T}) \cap S \Rightarrow T \cap S .
\end{aligned}
$$

These two covering sets of conditional rules are taken from the definitions of set membership and the intersection of two sets, respectively. Sets are represented as lists, so $H:: T$ is the set formed by adding element $H$ to the 
set $T$. Note that in the $\epsilon$ set only the second rule is a conditional wave-rule, but that in the $\cap$ set both rules are.

When applying conditional wave-rules CLAM proceeds as follows.

- If the condition is a variant of one of the existing hypotheses of the current case, then the rule can be applied with no further work.

- Else if the rule is a member of a covering set, then the current case is split into subcases using the conditions of that set.

- Otherwise, the condition is set up as an additional subgoal.

In this way we avoid dividing into subcases unless each of the subcases is likely to succeed. Since, for each subcase, there is a conditional rule whose condition is satisfied, then one of the following two situations will obtain.

- If the rule for a subcase is a conditional wave-rule, then rippling-out can continue.

- If the right-hand side of the rule for a subcase does not contain $\eta$, then the rule provides a non-recursive definition of $\eta$ in this case and the proof proceeds by symbolic evaluation (see [6]) rather than induction.

This gives some assurance of success in each subcase.

This is only a partial solution to the problems of using conditional rules. We do not invest much effort into proving the condition in the current case before giving up and dividing into cases. Thus, we will sometimes create an unnecessary case split.

To illustrate the use of conditional wave-rules consider the theorem:

$$
\begin{gathered}
\forall A: \text { nat. } \forall B: \text { set (nat). } \forall C: \text { set (nat). } \\
A \in B \wedge A \in C \rightarrow A \in B \cap C .
\end{gathered}
$$

The induction conclusion of this theorem is

$$
(a \in e:: \underline{b}) \wedge(a \in c) \rightarrow a \in(e:: \underline{b} \cap c) .
$$

Consider the second wave-front. Assume that the only matching wave-rules are the covering set for $\cap$ in Example 3. We can use this covering set to suggest a division into two cases, each of which has some assurance of success. After dividing into these two cases and applying the conditional wave-rules we get:

$$
\begin{aligned}
& e \in c \vdash a \in e: \underline{b} \wedge a \in c \rightarrow a \in e:: \underline{(b \cap c)}, \\
& \neg e \in c \vdash a \in e:: \underline{b} \wedge a \in c \rightarrow a \in b \cap c .
\end{aligned}
$$


The remaining wave-fronts can be rippled out once, each with the waverule

$$
X \in H:: \underline{T} \Rightarrow X=H \vee \underline{X \in T} .
$$

This gives

$$
\begin{aligned}
& e \in c \vdash((a=e \vee \underline{a \in b}) \wedge a \in c \rightarrow a=e \vee \underline{a \in b \cap c}, \\
& \neg e \in c \vdash(a=e \vee \underline{a \in b}) \wedge a \in c \rightarrow a \in b \cap c,
\end{aligned}
$$

and the wave-fronts introduced by this rule can be beached with various propositional wave-rules, e.g. the distributive law of $\wedge$ over $\vee$. The second case, for example, becomes:

$$
\neg e \in c \vdash \begin{aligned}
& (a=e \wedge a \in c \rightarrow a \in b \cap c) \wedge \\
& (a \in b \wedge a \in c \rightarrow a \in b \cap c)
\end{aligned}
$$

After strong fertilization this leaves the residue

$$
\neg e \in c \vdash(a=e \wedge a \in c \rightarrow a \in b \cap c) \wedge \text { true, }
$$

which can be readily proved. The first case is similar.

\section{Rippling-sideways into sinks}

Our fourth extension is to consider a third way in which rippling can successfully terminate. In our examples so far we have transformed universally quantified variables into skolem constants in both induction hypothesis and induction conclusion. However, universally quantified non-induction variables can be transformed into free variables in the induction hypothesis and skolem constants in the induction conclusion. These free variables are not constrained to be matched to their corresponding skolem constants during fertilization-they can be matched to any expressions.

This significantly increases the options open to the theorem prover. Wavefronts can be rippled sideways to surround the skolem constants, which we will call sinks. These sinks absorb the new wave-fronts and the engorged sinks can then be matched to the corresponding free variables during fertilization. In principle, this rippling-sideways can always be done using ordinary waverules: first applied forwards, then applied backwards. In practice, not all the required wave-rules are likely to be available, and the central part of the rippling must be done by a new kind of wave-rule. We will call these new kinds of wave-rule transverse wave-rules, and we will rename the original wave-rules as longitudinal wave-rules. This terminology of sideways, transverse, and longitudinal is natural in the graphical representation of rippling, cf. Fig. 4. 


\subsection{The format of transverse wave-rules}

A simple transverse wave-rule is a rewrite rule of the form

$$
\eta\left(\underline{\xi(\underline{\mu})}^{\dagger}, \nu\right) \Rightarrow \eta\left(\mu, \zeta(\underline{\nu}){ }^{\dagger}\right)
$$

i.e. it moves a wave-front from one argument of the function $\eta$ to another one. $\eta, \xi$, and $\zeta$ are each a non-empty term with a distinguished argument. If $\zeta$ were empty, then the rule would be a longitudinal wave-rule, cf. format (3) with $\zeta$ empty. Note the directional annotations on the wave-fronts. These reflect the fact that the purpose of applying a transverse wave-rule is to reverse the outwards direction of the original wave-front and send it inwards towards a sink.

Just as for longitudinal rules, the simple transverse wave form can be generalised, to give the multi-wave form

$$
\begin{aligned}
& \text { Cond } \rightarrow \eta\left(\xi_{1}\left(\underline{\mu_{1}}\right){ }^{\dagger}, \ldots, \xi_{n}\left(\underline{\left.\mu_{n}\right)}{ }^{\dagger}, \nu_{1}, \ldots, \nu_{m}\right)\right. \\
& \Rightarrow \eta\left(\mu_{1}, \ldots, \mu_{n}, \zeta_{1}\left(\underline{\nu_{1}}\right), \ldots, \zeta_{m}\left(\underline{\nu_{m}}\right)\right.
\end{aligned}
$$

although, we cannot generalise this to wave-fronts with multiple wave-holes or to multiple $\eta$ 's. A longitudinal wave-front would be required on the lefthand side to contain multiple $\eta$ 's. Without multiple $\eta$ 's we require a $1-1$ mapping between the $\mu$ 's and $\nu$ 's on the left- and right-hand sides, and this prevents multiple wave-holes. However, these restrictions are lifted when we consider hybrid transverse and longitudinal wave-rules in Section 8.

Note that each transverse wave-rule has a reverse dual which is also a transverse wave-rule. This reverse dual is formed by exchanging the lefthand side and right-hand side expressions and inverting the directional annotations, i.e. the reverse dual of format (32) above is

$$
\begin{aligned}
& \text { Cond } \rightarrow \eta\left(\mu_{1}, \ldots, \mu_{n},{\zeta \zeta_{1}\left(\underline{\nu_{1}}\right)}^{\uparrow}, \ldots, \zeta_{m}\left(\underline{\left.\nu_{m}\right)}{ }^{\uparrow}\right)\right. \\
& \Rightarrow \eta\left({\underline{\xi_{1}\left(\underline{\mu_{1}}\right)}}^{\downarrow}, \ldots, \xi_{n}\left(\underline{\left.\mu_{n}\right)}, \nu_{1}, \ldots, \nu_{m}\right)\right. \text {. }
\end{aligned}
$$

However, care must be taken if the original rule is irreversible, e.g. if it is based on implication or inequality (see Section 4.4 , third bullet). If an irreversible wave-rule can be applied to subexpressions of positive polarity, then its reverse dual can be applied to subexpressions of negative polarity, and vice versa. The use of both a transverse wave-rule and its reverse dual does not lead to looping because of the sense of direction imposed by the preconditions of rippling-sideways (see Section 6.3).

The preconditions for applying both conditional transverse wave-rules are the same as those for conditional longitudinal wave-rules (Definition 3) 
except that we must now check for the directional annotation on the wavefront.

Definition 4 (Preconditions of applying directional wave-rules). The preconditions of applying a directional wave-rule to rewrite a subexpression of the induction conclusion are that:

- the left-hand side of the rule matches the subexpression;

- the subexpression contains at least one wave-front;

- each wave-front in the subexpression is matched with a wave-front of the same kind in the rule; and

- the condition of the wave-rule is provable from the current hypotheses.

Some examples of transverse wave-rules are given below.

Example 4 (Transverse wave-rules).

$$
\begin{aligned}
& \left.(\underline{\underline{U}<>V}]^{\dagger}\right)<>W \Rightarrow U<>\left(\underline{V<>} \underline{W}^{\downarrow}\right), \\
& U<>\left(\underline{V<>\underline{W}}^{\dagger}\right) \Rightarrow\left(\underline{\underline{U}<>V}{ }^{\downarrow}\right)<>W, \\
& \left.\operatorname{qrev}(H d:: \underline{T l}]^{\uparrow}, L\right) \Rightarrow \operatorname{qrev}\left(T l, H d:: \underline{L}{ }^{\downarrow}\right), \\
& \operatorname{qrev}\left(T l, H_{H d:: \underline{\underline{L}}}^{\dagger}\right) \Rightarrow \operatorname{qrev}\left(H d:: \underline{T l}{ }^{\downarrow}, L\right) .
\end{aligned}
$$

Two pairs of transverse wave-rules are shown. The members of each pair are reverse duals. The first pair is formed from the associative law of list append, where $\langle>$ is infix append. This shows that associative laws can be interpreted as transverse wave-rules as well as longitudinal wave-rules (cf. rules (7) and (8) in Example 1). The second pair is formed from the step case of the tail-recursive definition of list reversal. Tail-recursive definitions which use accumulators are a major source of transverse wave-rules.

\subsection{A worked example}

To illustrate rippling-sideways, consider the following example. Let unary rev be the naive list reversal function and binary qrev be the tail recursive list reversal function, so that the following rewrite rules are available.

$$
\begin{aligned}
& \operatorname{rev}(\text { nil }) \Rightarrow \text { nil, } \\
& \operatorname{rev}\left(\underline{H d:: \underline{T l}}^{\dagger}\right) \Rightarrow \underline{\operatorname{rev}(T l)}<>(H d:: n i l){ }^{\dagger} ; \\
& \operatorname{qrev}(n i l, L) \Rightarrow L, \\
& \operatorname{qrev}\left({ }_{H d:: \underline{T l}}^{\dagger}, L\right) \Rightarrow \operatorname{qrev}\left(T l, H_{H d:: \underline{L}}^{\downarrow}\right) .
\end{aligned}
$$


The second argument of qrev is an accumulator. Note that (34) is a longitudinal wave-rule and (35) is a transverse wave-rule.

Consider the following theorem connecting these two list reversal functions.

$$
\forall L: \text { list (nat). } \forall M: l i s t(\text { nat }), \operatorname{rev}(L)<>M=\operatorname{qrev}(L, M) .
$$

To prove this theorem let $L$ be the induction variable. The other universally quantified variable, $M$, will become a skolem constant in the induction conclusion (represented by $m$ ) and a free variable in the induction hypothesis (represented by $M$ ). Note that when the induction hypothesis is used to fertilize the induction conclusion it is not necessary for $M$ to be instantiated to $m$. $M$ can take any value, provided all occurrences of $M$ are instantiated to identical values. This suggests a new way to prevent the wave-fronts from obstructing fertilization. They can be rippled sideways and inwards until they are absorbed by the sink $m$. Fertilization will then instantiate $M$ to $m$ and its surrounding wave-front. We will annotate the sink, $m$, with $\lfloor m\rfloor$, in order to signify that it is a target for wave-fronts.

So, by the discussion above, the induction hypothesis is

$$
\operatorname{rev}(l)<>M=\operatorname{qrev}(l, M),
$$

where $M$ is a free variable, and the induction conclusion is

$$
\left.\operatorname{rev}(h:: \underline{l}]^{\dagger}\right)<>\lfloor m\rfloor=\operatorname{qrev}\left(h:: \underline{l}^{\dagger},\lfloor m\rfloor\right) .
$$

To make the induction conclusion match the induction hypothesis we will ripple the two wave-fronts sideways so that each surrounds an $\lfloor m\rfloor$. Applying rule (35) does this to the right-hand side wave-front in one step.

$$
\left.\left.\operatorname{rev}\left(h:: \underline{l}^{\uparrow}\right)<>\lfloor m\rfloor=\operatorname{arev}(l, \mid h:: \underline{m}]^{\dagger}\right\rfloor\right) .
$$

We draw the sink annotation outside the wave-front to show that it has absorbed the wave-front.

Rippling the left-hand wave-front is more difficult. First, (34) is applied to ripple the wave-front out:

$$
\left(\underline{\operatorname{rev}(l)}<>(h:: n i l)^{\dagger}\right)<>\lfloor m\rfloor=\operatorname{qrev}\left(l,\left\lfloor h::\left.\underline{m}\right|^{\downarrow}\right\rfloor\right) .
$$

Second, wave-rule (33), the associativity of $\langle>$, is used to ripple the wavefront sideways to the left hand $\lfloor m\rfloor$ :

$$
\left.\left.\operatorname{rev}(l)<>\left(\lfloor(h:: n i l)<>\underline{m}]^{\downarrow}\right\rfloor\right)=\operatorname{qrev}\left(l,|h:: \underline{m}|^{\downarrow}\right\rfloor\right) .
$$

Third, normalisation is applied to simplify the wave-fronts in the sinks and make them identical. This rewrites $(h:: n i l) \ll m$ to $h:: m$ on the left-hand side. This permits strong fertilization to match the induction 
hypothesis, (36), to the induction conclusion, which instantiates $M$ to $h:: m$, and completes the step case.

\subsection{Control of rippling-sideways}

Rippling-sideways is controlled as follows. Initially, all wave-fronts are annotated as outward directed waves. When a transverse wave-rule is applied, the new wave-fronts are all annotated as inwards directed (cf. the general format of transverse wave-rules above). The wave-holes of each of these new inwards wave-fronts must contain a sink, otherwise the transverse rule application is not permitted. Only rippling-in is permitted to move these inward directed wave-fronts. The version of rippling-in used at this stage is controlled differently from the version described in Section 4. Instead of wave-fronts being directed in order to enable cancellation, they are directed towards the sinks in their wave-holes. Rippling-in terminates when all inward directed wave-fronts are absorbed into these sinks. The effect of this control is that wave-fronts are first rippled out, then sideways and then in towards sinks. A diagrammatic representation of a simple case of this process is given in Fig. 4. Finally, the engorged sinks are simplified to facilitate fertilization. For fertilization to succeed each occurrence of a universally quantified variable in the induction hypothesis must match against an identical sink. Simplifying wave-fronts can turn equal wave-fronts into identical ones, as in the third stage of the example above.

The strong direction imposed on rippling by the directional annotation of wave-fronts prevents looping. Once a wave-front has started to move towards a sink it cannot move backwards just because there is a rule available to so move it. This explains why the presence of both a transverse wave-rule and its reverse dual does not cause a loop. This is another example of how the use of wave annotations and the preconditions of rippling tame a potentially explosive set of rewrite rules.

However, the presence of both longitudinal and transverse wave-rules does introduce a new cause of branching into rippling. It is now possible to have a longitudinal and a transverse wave-rule both meet the preconditions of rippling, so that there is a genuine choice in the search space. Moreover, these choices do not lead to slight variants of the same proof, as in our previous examples of such choice. If they do both lead to proofs, they will be significantly different ones. An example of a pair of alternative wave-rules is:

$$
\begin{aligned}
& s(\underline{U}){ }^{\uparrow}+V \Rightarrow s(\underline{U+V}) \\
& s(\underline{U}){ }^{\dagger}+V \Rightarrow U+s(\underline{V})
\end{aligned}
$$

In Section 4 we described the use of longitudinal wave-rules applied 


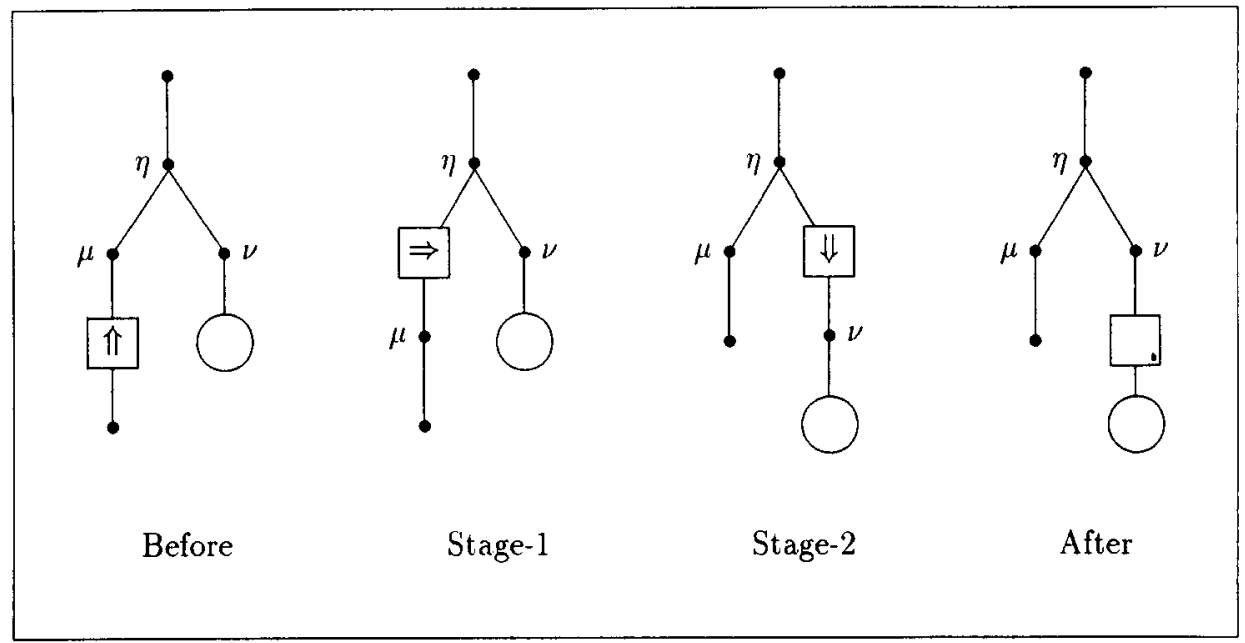

Fig. 4. Stages of transverse rippling. The four expression trees show the expression before, during and after rippling-sideways. Sinks are indicated by circle nodes and wave-fronts by square nodes, as in Fig. 3. $\eta$ is the least upper bound of the wave-front and sink. $\mu$ and $\nu$ are the immediate daughters of $\eta$ in the direction of the wave-front and sink, respectively. In Stage- 1 the wave-front has been rippled out until it is between the $\mu$ and $\eta$ nodes. In Stage-2 it has been rippled sideways so that it is between the $\nu$ and $\eta$ nodes. In After it has been rippled in to the sink.

backwards after weak fertilization to enable cancellation of identical functions from either side of an equation or similar formula. We called this rippling-in. There is an equivalent process for the backwards ${ }^{8}$ application of transverse wave-rules. Wave-fronts are rippled sideways out of sinks to enable cancellation. The control of this process is almost identical to that described in Section 4.4. An illustration of this process can be found in Section 11.2.2.

\subsection{Tail-recursion and transverse wave-rules}

We noted in Example 4 that the step cases of tail-recursive functions which use accumulators are a good source of transverse wave-rules. This is more than just a coincidence. The movement of a wave-front from recursion variable to accumulator is what defines the use of an accumulator to achieve tail-recursive behaviour. This definition then provides a wave-rule that moves a wave-front from induction variable to sink. Each occurrence of an accumulator provides a sink. Moreover, the verification, synthesis, or transformation of functions using accumulators usually requires ripplingsideways during any inductive proofs. Otherwise, the function's recursive

\footnotetext{
${ }^{8}$ Except note that an inverted transverse wave-rule is still a transverse wave-rule, so this is a another kind of ripple-sideways.
} 
definition cannot be used in the proof. The qrev example above illustrates this.

\section{Rippling-across and destructor inductions}

Our fifth extension is to adapt rippling to proofs by destructor induction. In all the proofs considered so far the induction rules have been in the constructor style, that is the induction hypothesis has been of the form $\phi(\chi)$ and the induction conclusion has been of the form $\phi(c(X))$, for some constructor function, $c$. This neglects proofs in the destructor style of induction, that is where the induction conclusion has the form $\phi(\chi)$ and there are one or more induction hypotheses, each of the form $\phi(d(\chi))$ for some destructor function, $d$. Many functions can only be defined using a destructor style of recursion, e.g. quicksort, and for these the destructor style of induction is usually more natural.

One approach to rippling in destructor induction proofs is to ripple forwards on the induction hypotheses rather than rippling backwards on the induction conclusion. We have attempted this approach. It is described in [9]. The difficulty is in using a rippled induction hypothesis, $\zeta(\phi(\chi))$, to prove the induction conclusion, $\phi(\chi)$. Unless $\zeta$ is empty, no fertilization is possible, so the overall inductive proof plan breaks down.

In this paper we take another approach-we ripple the wave-front across from the hypotheses to the conclusion, and then ripple the induction conclusion as before. This rippling-across consists of two stages:

- Creation. A new kind of wave-rule is applied to the induction conclusion which creates a wave-front and some anti-wave-fronts, where none of these wave-fronts was present before. Anti-wave-fronts are wave-fronts annotated with a "-" sign instead of a " $\uparrow$ " or " $\downarrow$ ". We call this new kind of rule a creational rule. Some examples of creational rules are given in Example 5. A condition of creational rule application is that each new anti-wave-front in the induction conclusion must correspond to an old wave-front in the induction hypotheses. By "correspond" we mean that not only must the wave-front and anti-wave-front match, but they must occur at the same relative position in their respective expressions.

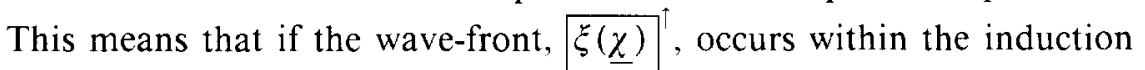
hypothesis,

$$
\left.\phi\left(\eta(\underline{\xi(\underline{\chi})}]^{\uparrow}\right)\right)
$$

then the creational rule must have the form

$$
\left.\eta(\nu) \Rightarrow \zeta\left(\eta(\xi(\nu)]^{-}\right)\right)
$$


- Neutralisation. The annotations on the anti-wave-fronts and their corresponding old wave-fronts in the induction hypotheses are both removed. These (anti-)wave-fronts are said to be neutralised.

The general format of creational rules is:

$$
\begin{aligned}
& \text { Cond } \rightarrow \eta\left(\nu_{1}, \ldots, \nu_{m}\right) \\
& \Rightarrow \frac{\zeta\left(\eta\left({\zeta \zeta_{1}^{1}\left(\underline{\nu_{1}}\right)}^{-}, \ldots,{\zeta \zeta_{m}^{1}\left(\nu_{m}\right)}^{-}\right),\right.}{\left.\ldots, \eta\left({\zeta \zeta_{1}^{k}\left(\underline{\nu_{1}}\right)}^{-}, \ldots,{\zeta \zeta_{m}^{k}\left(\nu_{m}\right)}^{-}\right)\right)}
\end{aligned}
$$

where Cond is a formula and $\eta$, the $\zeta_{i}^{j}$,s, and $\zeta$ are terms with distinguished arguments. $\zeta$ may be empty, but the $\zeta_{i}^{j}$ s s and $\eta$ must not be. The $\zeta_{i}^{j}$ 's are the new anti-wave-fronts and $\zeta$ is the new wave-front.

The preconditions for applying creational wave-rules require two new items in addition to those for conditional wave-rules (Definition 4 ). We postpone giving these until Section 8, Definition 5, where the final set of preconditions is given for a general form of wave-rule.

Example 5 (Creational wave rules).

$$
\begin{aligned}
& U \neq 0 \rightarrow U \Rightarrow s\left(\underline{\underline{p(\underline{U})}}{ }^{-},\right. \\
& U \neq 0 \rightarrow U+V \Rightarrow s\left(\underline{\underline{p(\underline{U}}-V)}{ }^{\top},\right. \\
& x \neq \text { nil } \rightarrow \\
& \text { conscells }(U)
\end{aligned}
$$

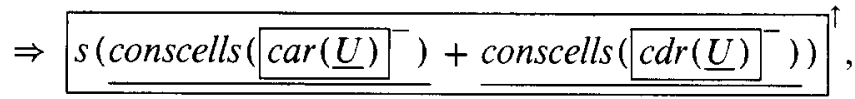

$$
\begin{aligned}
& x \neq \operatorname{nil} \rightarrow \operatorname{len}(U) \Rightarrow s\left(\underline{\left.\left.\operatorname{len}(\underline{\operatorname{cdr}(\underline{U})})^{-}\right)\right)} .\right.
\end{aligned}
$$

Creational rules are mainly extracted from destructor-style recursive definitions. For instance, the last three rules above are extracted from the destructor-style recursive definitions of + , conscells, and len, respectively. The first rule is from a lemma about $s$ and $p$. 


\subsection{A simple example}

Here is an example destructor induction step case from the proof of the associativity of + . The destructor-style definition of + is as follows:

$$
\begin{aligned}
U+V= & \text { if } U=0 \text { then } V \\
& \text { else } s(p(U)+V)
\end{aligned}
$$

From this definition we can readily extract the following creational rule:

$$
U \neq 0 \rightarrow U+V \Rightarrow s(\underline{\underline{p(\underline{U})}+V)},
$$

which is used twice in the first step of the proof as follows (the induction hypothesis is to the left of the $\vdash$ and the induction conclusion is to its right):

$$
\begin{aligned}
& p(\underline{x}){ }^{\dagger}+(y+z)=\left(p(\underline{x}){ }^{\dagger}+y\right)+z \\
& \vdash x+(y+z)=(x+y)+z, \\
& \underline{p(\underline{x})}]^{\uparrow}+(y+z)=\left(\underline{p(\underline{x})}{ }^{\uparrow}+y\right)+z \\
& \vdash s\left(\underline{\underline{p(\underline{x})}-(y+z))}{ }^{-}=s(\underline{\underline{p(\underline{x})}+y)}+z,\right. \\
& p(x)+(y+z)=(p(x)+y)+z \\
& \vdash s(\underline{p(x)+(y+z)})^{\dagger}=s(\underline{p(x)+y)}+z .
\end{aligned}
$$

The last step is not an object-level inference, but the purely meta-level neutralisation of the (anti-) wave-fronts.

\subsection{The application of creational wave-rules}

Rippling-across is applied immediately after any application of destructor style induction. It rewrites the induction conclusion into a form to which the other forms of rippling are applicable and neutralises wave-fronts in the induction hypotheses. Each of the wave-fronts in each induction hypothesis is considered in turn. Let $\mathcal{C}$ be the set of creational rules with corresponding anti-wave-fronts.

If $\mathcal{C}$ is empty for some wave-front in some induction hypothesis, then this hypothesis is unusable, so it is labelled "unviable" and is not considered further. Otherwise, each of the rules in $\mathcal{C}$ is considered in turn. The subexpression, $\eta(\chi)$, in the induction conclusion, $\phi(\eta(\chi))$, is rewritten with this rule. Each of the newly introduced anti-wave-fronts and their corresponding wave-fronts in viable induction hypotheses are neutralised. Note that we attempt to neutralise all new anti-wave-fronts and not just the one that triggered the rule application. Note also that corresponding wave-fronts in 
several induction hypotheses may be neutralised and not just the one that triggered the rule application. Note finally that induction hypotheses may be discovered to be unviable after an anti-wave-front has been neutralised with one of its wave-fronts. This neutralisation must be undone, and another way must be sought to neutralise the anti-wave-front.

This process succeeds if at least one induction hypothesis remains viable and all its wave-fronts are neutralised. If any anti-wave-front in the induction conclusion remains unneutralised, then it is merged with the wave-front that immediately surrounds it. This merging process is identical to that used in the weakening of wave-rules (see Section 3.4).

\subsection{A more complex example}

To illustrate this process, consider the following example, which has been chosen to exhibit all the various complications. The theorem to be proved is

$$
\forall L: l i s t \text { (nat). conscells }(L) \geqslant \operatorname{len}(L),
$$

where conscells counts the number of cons cells used in a list and len counts its length. Suppose we try to prove this with a $c a r / c d r$ destructor induction on lists. The step case will be:

$$
\begin{aligned}
& l \neq n i l \text {, } \\
& \text { conscells }\left(\operatorname{car}(\underline{l})^{\uparrow}\right) \geqslant \operatorname{len}\left(\operatorname{car}(\underline{l})^{\dagger}\right) \text {, } \\
& \text { conscells }\left(c d r(\underline{l}){ }^{\dagger}\right) \geqslant \operatorname{len}\left(c d r(\underline{l}){ }^{\dagger}\right) \\
& \vdash \text { conscells }(l) \geqslant \operatorname{len}(l) \text {. }
\end{aligned}
$$

Note that there are two induction hypotheses. We will refer to them as the car induction hypothesis and the $c d r$ induction hypothesis, respectively.

The only relevant creational rules are

$$
\begin{aligned}
& x \neq \text { nil } \rightarrow \\
& \text { conscells }(U) \\
& \Rightarrow{\text { s(conscells } \left.\left.(\underline{\operatorname{car}(\underline{U})})^{-}\right)+\operatorname{conscells}\left(\underline{\operatorname{cdr}(\underline{\underline{U}})}{ }^{-}\right)\right)}^{\top}, \\
& x \neq \text { nil } \rightarrow \operatorname{len}(U) \Rightarrow s\left(\underline{\left.\operatorname{len}(\underline{c d r(\underline{U})})^{-}\right)} .\right.
\end{aligned}
$$

We consider, in turn, each of the wave-fronts in each of the induction hypotheses. Consider the first wave-front of the car induction hypothesis. 
The conscells rule has a corresponding anti-wave-front. Applying it produces:

$$
\begin{aligned}
& l \neq n i l, \\
& \text { conscells }\left(\operatorname{car}(\underline{l})^{\dagger}\right) \geqslant \operatorname{len}\left(\operatorname{car}(\underline{l})^{\dagger}\right) \text {, } \\
& \text { conscells }\left(\operatorname{cdr}(\underline{l})^{\dagger}\right) \geqslant \operatorname{len}\left(c d r(\underline{l}){ }^{\dagger}\right)
\end{aligned}
$$

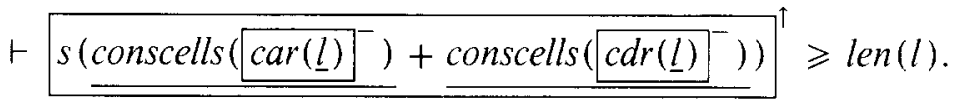

Each of the two new anti-wave-fronts neutralises with a corresponding wavefront in one of the two induction hypotheses.

$$
\begin{aligned}
& l \neq \text { nil }, \\
& \text { conscells }(\operatorname{car}(l)) \geqslant \operatorname{len}\left(\underline{\operatorname{car}(\underline{l})}^{\dagger}\right), \\
& \text { conscells }(\operatorname{cdr}(l)) \geqslant \operatorname{len}\left(\underline{\operatorname{cdr}(\underline{l})}{ }^{\dagger}\right) \\
& \quad \vdash \underline{\text { s conscells }(\operatorname{car}(l))+\operatorname{conscells}(\operatorname{cdr}(l)))}{ }^{\dagger} \geqslant \operatorname{len}(l) .
\end{aligned}
$$

Now consider the second wave-front of the car induction hypothesis. Note that neither of the creational rules contains an anti-wave-front corresponding to this wave-front, so the car induction hypothesis is labeled "unviable" and takes no further part in the proof. We will delete it. The car anti-wave-front created by the conscells rule was neutralised by a wave-front in this unviable induction hypothesis. Since this car anti-wave-front cannot be neutralised in any other way in must be reinstated. So the current proof state is:

$$
\begin{aligned}
& l \neq \text { nil }, \\
& \text { conscells }(\operatorname{cdr}(l)) \geqslant \operatorname{len}\left(\left[{\left.\operatorname{cdr}(\underline{l})]^{\dagger}\right)}^{\left.\operatorname{s(\text {conscells}([\operatorname {car}(\underline {l})^{-})}+\operatorname{conscells}(\operatorname{cdr}(l))\right)}{ }^{\uparrow} \geqslant \operatorname{len}(l) .\right.\right.
\end{aligned}
$$

Now consider the second wave-front of the $c d r$ induction hypothesis. The len rule has a corresponding anti-wave-front. Applying it and neutralising the corresponding wave- and anti-wave-fronts produces

$$
\begin{aligned}
& l \neq n i l, \\
& \text { conscells }(c d r(l)) \geqslant \operatorname{len}(\operatorname{cdr}(l)) \\
& \left.\vdash s\left(\text { conscells }\left(\operatorname{car}(l)^{-}\right)\right)+\text {conscells }(\operatorname{cdr}(l))\right) \\
& \geqslant s^{(\operatorname{len}(c d r(l)))}{ }^{\uparrow} \text {. }
\end{aligned}
$$


The induction conclusion now contains an unneutralised anti-wave-front. This must be merged with the wave-front that immediately surrounds it, to produce

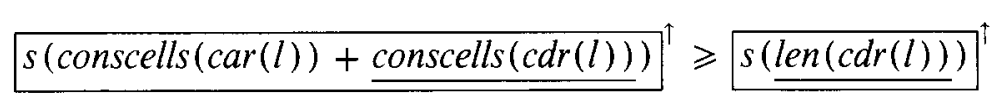

Constructor-style rippling can now be brought to bear on the induction conclusion using the longitudinal wave-rules:

$$
\begin{aligned}
& s(\underline{U}){ }^{\uparrow} \geqslant s(\underline{V}){ }^{\uparrow} \Rightarrow U \geqslant V, \\
& U+\underline{V}{ }^{\uparrow} \geqslant W \Rightarrow V \geqslant W .
\end{aligned}
$$

To apply these rules it is necessary first to split the left-hand side wave-front,

$$
\begin{aligned}
& s\left(\underline{\text { conscells }(\text { car }(l))+\text { conscells }(\text { cdr }(l))}{ }^{\dagger}\right) \\
& \geqslant s\left(\underline{\operatorname{len}(c d r(l)))}{ }^{\uparrow}\right.
\end{aligned}
$$

and ripple each wave-front out separately. Strong fertilization then applies, completing the step case.

\section{A general format for wave-rules}

We have now introduced three different kinds of wave-rule-longitudinal, transverse, and creational-each of which can also be conditional. In the search for more power we have lost some of the original simplicity of rippling. There seems to be a danger that this complexity will grow indefinitely. In this section we try to reduce the complexity by defining a general format which includes all three kinds of wave-rule.

\subsection{Combining the formats}

As a starting point we repeat below the forms of the three different kinds of wave-rules:

- Longitudinal:

$$
\begin{aligned}
& \text { Cond } \rightarrow \\
& \qquad \begin{array}{l}
\eta\left(\underline{\xi_{1}\left(\underline{\mu_{1}^{1}}, \ldots, \underline{\mu_{1}^{p_{1}}}\right){ }^{\uparrow}, \ldots, \xi_{n}\left(\underline{\mu_{n}^{1}}, \ldots, \underline{\left.\mu_{n}^{p_{n}}\right)}{ }^{\dagger}\right)}\right. \\
\quad \Rightarrow \zeta\left(\underline{\left.\eta\left(\varpi_{1}^{1}, \ldots, \varpi_{n}^{1}\right), \ldots, \eta\left(\varpi_{1}^{k}, \ldots, \varpi_{n}^{k}\right)\right)} ;\right.
\end{array}
\end{aligned}
$$


- Transverse:

Cond $\rightarrow$

$$
\begin{aligned}
& \eta\left(\xi_{1}\left(\mu_{1}\right){ }^{\uparrow}, \ldots, \xi_{n}\left(\mu_{n}\right){ }^{\uparrow}, \nu_{1}, \ldots, \nu_{m}\right) \\
& \Rightarrow \eta\left(\mu_{1}, \ldots, \mu_{n}, \zeta_{1}\left(\underline{\nu_{1}}\right)\right.
\end{aligned}
$$

- Creational:

Cond $\rightarrow$

$$
\begin{aligned}
& \eta\left(\nu_{1}, \ldots, \nu_{m}\right)
\end{aligned}
$$

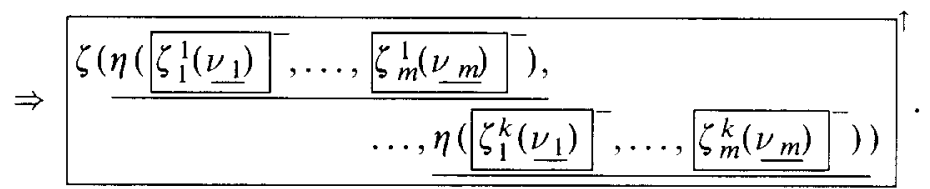

These three formats can be generalised to

$$
\begin{aligned}
& \text { Cond } \rightarrow \\
& \eta\left(\xi_{1}\left(\underline{\mu_{1}^{1}}, \ldots, \underline{\mu_{1}^{p_{1}}}\right){ }^{\uparrow}, \ldots, \xi_{n}\left(\underline{\underline{\mu_{n}^{1}}, \ldots, \underline{\left.\mu_{n}^{p_{n}}\right)}}{ }^{\uparrow}, \nu_{1}, \ldots, \nu_{l}, \nu_{l+1}, \ldots, \nu_{m}\right)\right.
\end{aligned}
$$

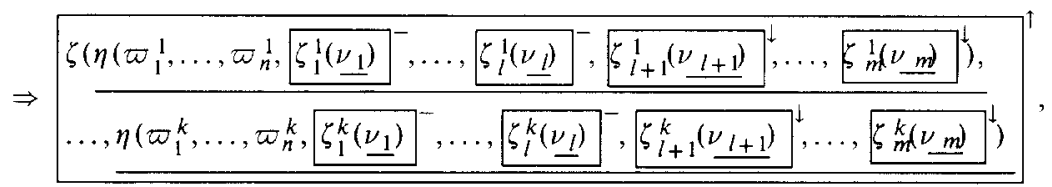

where each $\varpi_{i}^{j}$ is either an unrippled wave-front,

$$
\xi_{i} \underline{\left.\underline{\mu_{i}^{1}}, \ldots, \underline{\mu_{i}^{p_{i}}}\right)}
$$

or is one of the wave-holes, $\mu_{i}^{l}$. For each $j$, at least one $\varpi_{i}^{l}$ must be a wave-hole. Cond is a formula. $\eta$, the $\xi_{i}$ s, $\zeta$, and the $\zeta_{i}^{j}$ s are terms with distinguished arguments. $\zeta$ may be empty, but the $\xi_{i}$ 's, $\zeta_{i}^{j}$ 's and $\eta$ must not be. For notational convenience, but without loss of generality, we have ordered the arguments of $\eta$ so that the outwards wave-fronts are the first $n$, the anti-waves are the next $l$ and the inwards wave-fronts are the last $m-l$. Either $n, m$, or $l$ may be 0 . If $m=0$, then the rule is purely longitudinal. If $n=0$ and $l=m$, then the rule is purely creational. If $\zeta$ is empty and $l=0$, then $p_{i}=1$ and the rule is purely transverse.

This generalised form also increases the power of rippling, since it allows hybrid wave-rules, e.g. rules that are partly longitudinal and partly transverse, 
for instance

$$
\sum_{i=0}^{s(\underline{n})} u_{i} \Rightarrow u_{0}+\sum_{\underline{i=0} u^{s(\underline{i})}}^{n},
$$

or partly longitudinal and partly creational, for instance

$$
\begin{aligned}
& \text { quicksort }\left(h d:: \underline{t l}{ }^{\uparrow}\right)
\end{aligned}
$$

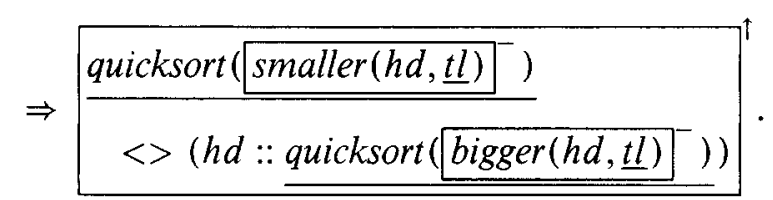

Rippling-out, rippling-sideways, and rippling-across are combined in CLAM as the selective application of wave-rules in the above general format. The wave annotations determine what sort of rippling takes place, with hybrid forms of rippling happening in a natural way. In practice, all $\zeta_{i}^{j}$ wave-fronts are labeled as inward directed. Whether they are treated as anti-wave-fronts depends on whether there is a wave-front to be neutralised in the induction hypothesis.

\subsection{Rippling-in}

Rippling-in applies general wave-rules from right to left. When used for rippling-in, the wave-front directional annotations are different from that given above. So rippling-in applies-left to right-waves of the form

Cond $\rightarrow$

$$
\begin{aligned}
& \frac{\zeta\left(\eta\left(\varpi_{1}^{1}, \ldots, \varpi_{n}^{1}, \zeta_{1}^{1}\left(\nu_{1}\right), \ldots, \underline{\zeta_{m}^{1}\left(\nu_{m}\right)}\right), \ldots,\right.}{\left.\underline{\eta\left(\varpi_{1}^{k}, \ldots, \varpi_{n}^{k}, \zeta_{1}^{k}\left(\underline{\nu_{1}}\right), \ldots, \zeta_{m}^{k}\left(\underline{\nu_{m}}\right)\right.}\right)} \\
& \Rightarrow \eta\left(\xi_{1}\left(\underline{\mu_{1}^{1}}, \ldots, \underline{\mu_{1}^{p_{1}}}\right), \ldots, \xi_{n}\left(\underline{\mu_{n}^{1}}, \ldots, \mu_{n}^{p_{n}}\right), \nu_{1}, \ldots, \nu_{m}\right)
\end{aligned}
$$

where the range of the symbols is as before, except that $\zeta$ must not be empty, since purely transverse rules are not used for rippling-in. The direction of the $\zeta_{i}^{j}$ wave-fronts can be either inwards or outwards.

\subsection{Applying generalised rippling}

We have implemented rippling as a single tactic that applies general wave-rules left to right or right to left. It analyses the current induction conclusion and chooses a rule which will move the wave-fronts in a desirable 
direction while keeping the skeleton intact. This generalised ripple tactic embodies, as special cases, rippling-out, rippling-sideways, rippling-across, and rippling-in. Despite the complications of applying wave-rules in both left/right orientations, the duplication of skeletons, the choice of directions to ripple in, etc., the ripple tactic always terminates. This is proved in Appendix A.

The preconditions of generalised rippling are the same as those for conditional rippling (Definition 3), but with two additional items for dealing with creational rippling.

Definition 5 (Preconditions of applying generalised wave-rules). The preconditions of applying a generalised wave-rule to rewrite a subexpression of the induction conclusion are that:

- the left-hand side of the rule matches the subexpression;

- the subexpression contains at least one wave-front;

- each wave-front in the subexpression is matched with a wave-front of the same kind in the rule;

- if the wave-rule contains an anti-wave-front, then at least one neutralisation must occur.

- after neutralisation at least one viable induction hypothesis remains;

- after merging unneutralised anti-wave-fronts there exists at least one instance of the skeleton in the new induction conclusion; and

- the condition of the wave-rule is provable from the current hypotheses.

The generalised ripple tactic does not embody our last extension: existential rippling. Nor is existential rippling guaranteed to terminate. This form of rippling extends generalised rippling and is still under development. We describe its current state in the next section.

\section{Rippling under existential quantifiers}

The ripple tactic developed so far is suitable for proving theorems containing universal quantifiers, but it cannot cope with existential quantifiers. The problem is that the witness of an existential quantifier, i.e. the term that the quantifier asserts to exist, may well contain occurrences of the induction variable. In this case the witness of the existential quantifier in the induction conclusion will contain wave-fronts and must take part in rippling. Until the identity of the witness is known this rippling cannot be completed. On the other hand, we would like to use the possibilities for successful rippling to help us determine what the witness should be-a Catch-22 situation.

To illustrate the problem consider the following theorem.

$$
\forall X: \text { nat. } \forall Y: \text { nat }: \forall Z: \text { nat. } \exists W: \text { nat. } X+(Y+Z)=W+Z,
$$


where the witness for $W$ is clearly going to be $X+Y$, but we don't know that yet. If we apply $s(X)$ induction, then the induction conclusion is:

$$
\forall Y: n a t . \forall Z: n a t . \exists W: \text { nat. } s(\underline{x})+(Y+Z)=W+Z .
$$

This can be rippled to

$$
\forall Y: \text { nat. } \forall Z: \text { nat. } \exists W: \text { nat. } s(\underline{x+(Y+Z))}=W+Z,
$$

but no further rippling is possible.

In the purely universal version of this theorem, namely the associativity of + , we would ripple the right-hand side into the form $s(\ldots)$, and then ripple both $s$ functions away together. The right-hand side $s$ function would be put in place by an application of wave-rule (4), reproduced below

$$
s(\underline{U})+V \Rightarrow s(\underline{U+V}) \text {, }
$$

but this rule is not applicable unless $W$ is instantiated to something of the form $s(\ldots)$. One solution to this problem is to create an existential version of the wave-rule (4) and apply this instead, namely,

$$
\begin{aligned}
& \exists U^{\prime} \text { :nat. } s(x+(y+z))=U^{\prime}+V \\
& \Rightarrow \exists U \text { Unat. } s(x+(y+z))=s(\underline{U}+V) \text {. }
\end{aligned}
$$

Note that $U^{\prime}$ is marked as a wave-front because it is implicitly $s(\underline{U})$. For the application of this rule to (37) to qualify as rippling, $W$ must also be seen, as a wave-front. This is legitimate because, as we have seen, it might be instantiated to a term containing a wave-front. We therefore regard all existentially quantified variables in goals as potential wave-fronts, which we mark as $W$. The same argument holds for $U$ in (38). So applying (38) to (37) gives

$$
\forall Y: n a t . \forall Z: n a t . \exists: W_{1} \text { nat. } s(x+(Y+Z))=s\left(\underline{\left.\left.W_{1}+Z\right)\right)},\right.
$$

which can be rippled to

$$
\forall Y: n a t . \forall Z: n a t . \exists\left[\begin{array}{l}
W_{1} \\
0
\end{array} \text { at. } x+(Y+Z)=\left(\begin{array}{l}
W_{1} \\
\hdashline
\end{array}\right.\right.
$$

to which strong fertilization applies, completing the step case of the proof.

In this proof the relationship between $U$ and $U^{\prime}$ in wave-rule (38), and hence that between $W$ and $W_{1}$ in the induction conclusion, is not explicitly recorded. This does not matter if we are just interested in provability, but it does if we are also interested in the identity of the existential witness. The main application of our research is to program synthesis [5], in which the existential witnesses play the vital role of defining the programs to be synthesised. Fortunately, the logic we use-a variant of Martin-Löf type 
theory-also contains a solution to the problem. Each rule of inference of the logic has an associated program construction rule. A partial program is associated with each formula of the proof and is incrementally constructed in the course of the proof. The relationship between $U$ and $U^{\prime}$ in wave-rule (38) is contained in the program associated with its proof from wave-rule (4). The program associated with the step case of our example is a recursion equation partially defining a function, say $w$, which can serve as the witness of our existential quantifier, namely,

$$
w(s(X), Y, Z)=s(w(X, Y, Z)) .
$$

The program associated with the base case will be

$$
w(0, Y, Z)=Y,
$$

so that $w(X, Y, Z)=X+Y$.

Existential wave-rules are generated dynamically by CLAM, from regular wave-rules, according to need. This is essential since each regular wave-rule can give rise to infinitely many existential wave-rules. In the general case, the regular wave-rule

$$
\begin{aligned}
& \text { Cond } \rightarrow
\end{aligned}
$$

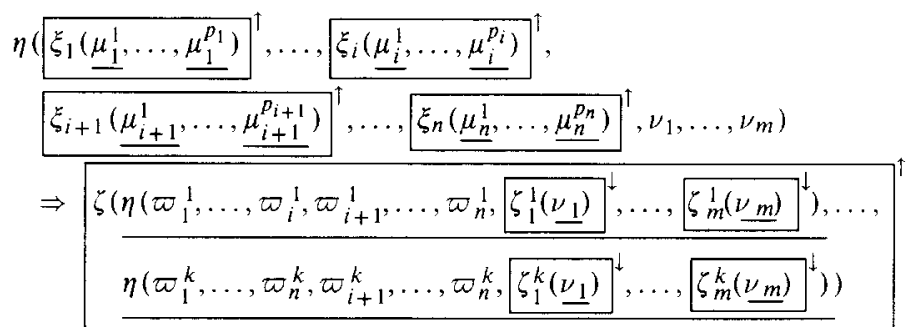

can be made into the existential rule

$$
\begin{aligned}
& \text { Cond } \rightarrow
\end{aligned}
$$

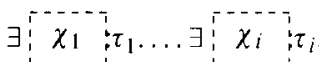

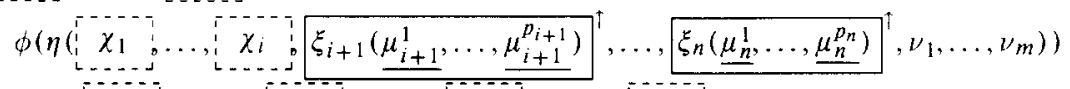

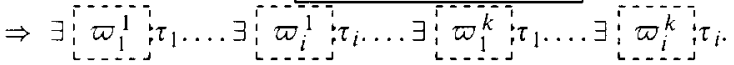

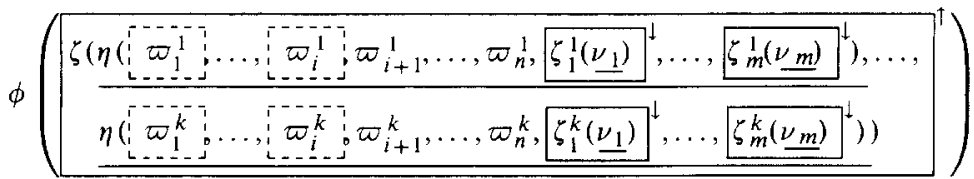

where $\phi$ is an arbitrary formula. Since there are infinitely many such $\phi$ this can be done in infinitely many ways, but we will see that, in practice, we can uniquely identify the $\phi$ we want. 
For notational convenience, but without loss of generality, we have ordered the arguments of $\eta$ so that those to be existentially quantified are the first $i$. Note that the $\varpi_{q}$ and $\varpi_{j}^{q}$ must all be variables. Any non-empty subset of the $n$ wave-fronts of $\eta$ can be chosen to be existentially quantified, i.e. it can be done in $2^{n}-1$ ways, but again, in practice, the appropriate subset can be uniquely determined.

Each side of the regular wave-rule is surrounded by a formula $\phi$, so that the existential quantifiers can be applied to a formula, rather than a term. CLAM chooses $\phi$, and the subset of wave-fronts to be existentially quantified, dynamically by inspection of the formula to be rewritten. For instance, in wave-rule (38), $\phi(\cdots)$ is chosen to be $s(x+(y+z))=\cdots$.

The presence of the potential wave-fronts around the $\mu_{j}^{q}$ means that the well-founded measure, introduced in Appendix A, might not be decreased by an existential rule application. Thus rippling under existential quantifiers is not guaranteed to terminate. One way to see this is that until the identity of the existential witnesses is fixed it is not clear how much rippling we will need to do. Since we can add to the complexity of these witnesses during the construction of the proof, the amount of rippling required is unbounded. We can avoid getting trapped into branches in which potential wave-fronts are merely continually instantiated by using the heuristic of preferring to ripple real wave-fronts whenever possible.

Work on existential rippling is still in progress. We have an initial implementation and have successfully tested it on a few simple examples. The problems of search are clearly exacerbated by the presence of existential quantifiers, but more empirical work is required to assess the size of the problem and extent to which existential rippling cures it.

\section{Implementation}

All the various forms of rippling described above have been implemented and tested in the Oyster/CLAM system [8], which is written in Prolog. Versions for Quintus and SICStus Prolog exist. A ripple tactic has been built. A tactic is a Prolog program which applies rules of inference of the Oyster type theory. The ripple tactic forms part of the indstrat tactic, which controls the whole of an application of inductive inference. Within indstrat, ripple is applied during the step case of an induction just after the induction tactic and before the fertilize tactic (see Fig. 2). The ripple tactic calls the wave subtactic, which applies individual wave-rules. Each tactic has a corresponding method, which specifies its preconditions and effects using a meta-logic implemented as a Prolog program. For instance, the precondition of the wave method is a Prolog program representing the preconditions given in English in Definition 5. These methods are used by CLAM to build 
a proof plan customised for the current theorem. Most proof plans are built from indstrat and a few other high-level tactics; their subtactics are only rarely used independently. More details of the Oyster/CLAM system can be found in [6]. ${ }^{9}$

In order to ensure the correct, selective application of wave-rules by ripple it is necessary to mark wave-fronts and wave-holes in both goals and wave-rules. CIAM does this by annotating the object-level expressions with meta-level functions. The Prolog functions wave_front/3 and wave_hole/ 1 are used to indicate wave-fronts and wave-holes, respectively. The arguments of wave_front are:

(1) the type of wave-front: definite or potential;

(2) the direction of rippling: out or in; and

(3) the object-level expression itself.

The in argument is used to indicate both inwards directed and anti-wavefronts. The argument of wave hole is just the object-level expression. Thus the expression $S(\underline{x}){ }^{\uparrow}+y$ is represented by the Prolog expression

wave_front (definite, out, $\mathrm{s}($ wave_hole $(\mathrm{x}))$ ) $+\mathrm{y}$.

This representational technique has the advantage that alignment of wavefronts in wave-rules and goals is automatically checked as a side-effect of the process of matching rule to goal.

Whenever a new definition is made or a new theorem proved, CLAM analyses it and extracts any wave-rules that it suggests. If necessary, these rules are proved as lemmas from the original definition or theorem. The wave-rules are automatically annotated with wave-fronts, possibly in multiple ways and in different left/right orientations. These multiple ways include weakenings, reverse duals, and both transverse and longitudinal readings of the same formula (cf. the many different ways in which the associativity laws can be regarded as wave-rules, see Examples 1 and 4). They are then stored for future use. The rippling capabilities of CLAM thus increase with use. Although the search space also increases this is not a problem in practice because the strong preconditions of rippling tame the combinatorial explosion.

\footnotetext{
${ }^{9}$ The Oyster-CLAM system is available either via anonymous FTP or on a tape cartridge. For further details please contact Gordon Reid, Department of Artificial Intelligence, University of Edinburgh, 80 South Bridge, Edinburgh, EH1 1HN, Scotland, UK. Telephone: $(+44-31) 650$ 2728. E-mail: gordon@uk.ac.ed.aisb.
} 


\section{Results}

The major hypothesis advanced in this paper is that in a significant number of inductive proofs the ripple tactic alone will successfully guide the step case between the application of the inductive rule to the use of the induction hypothesis. We predict that we can use just the ripple tactic in place of the mixture of heuristics used by other inductive theorem provers for this stage of inductive proofs, with similar or improved success. For instance, in the Boyer-Moore theorem prover, Nqthm, this stage of the proof is controlled by the heuristics of rewriting terms; eliminating destructors; using definitions, axioms and lemmas; and by the user giving hints. We predict that rippling will successfully replace all of these giving a more uniform search control regime and will prove some theorems that Nqthm cannot prove without user guidance.

To test this prediction we have used rippling to control the search of a large number of theorems drawn from the literature. Below we give statistics on its performance which confirm our prediction. We then give a number of selected examples to show how rippling generalises and replaces the heuristics used in other theorem provers and how it can guide steps which in other systems must be controlled by human intervention.

\subsection{Quantitative study}

We have tested rippling by running CLAM successfully on 82 simple inductive theorems drawn from Boyer and Moore [3], Kanamori and Fujita [16], and other standard sources. In each proof, the ripple tactic is solely responsible for controlling the proof between the application of induction and the application of fertilization, i.e. rippling alone puts the induction conclusion into a form in which it can be simplified with the aid of the induction hypothesis.

A selection of the theorems proved by CLAM is listed below.

Example 6 (Sample theorems proved using rippling).

$$
\begin{aligned}
& \forall X: \text { nat. } \forall Y: \text { nat. } \forall Z: \text { nat. } X+(Y+Z)=(X+Y)+Z, \\
& \forall L: \text { list (int). } \forall M: l i s t(\text { int }) . \operatorname{rev}(L)<>M=\operatorname{qrev}(L, M), \\
& \forall A: \operatorname{int} . \forall L: \operatorname{list}(\text { int }) . \operatorname{count}(A, \operatorname{sort}(L))=\operatorname{count}(A, L), \\
& \forall L: \text { list }(\text { int }) \text {. length }(\operatorname{sort}(l))=\text { length }(l), \\
& \forall X: \text { int. } \forall N: \text { nat. } \forall L: \text { list(int). } X \in n t h(N, L) \rightarrow X \in L, \\
& \forall X: n a t . \forall Y: n a t . e v e n(X) \wedge \operatorname{even}(Y) \rightarrow \operatorname{even}(X+Y)
\end{aligned}
$$


Table 1

Kinds of rippling used in proofs. Each row of the table shows the kinds of rippling used by CIAMto prove a theorem in Example 6. The first entry gives the number of the theorem and the subsequent entries give the kinds of rippling used in its proof.

\begin{tabular}{|c|c|c|c|c|}
\hline Theorem number & Out & Sideways & In Across & Existential \\
\hline$(39)$ & $\mathrm{x}$ & & & \\
\hline$(40)$ & $\mathrm{x}$ & $\mathrm{x}$ & & \\
\hline (41) & $\mathrm{x}$ & & & \\
\hline (42) & $\mathrm{x}$ & & & \\
\hline (43) & $\mathrm{x}$ & $\mathrm{x}$ & & \\
\hline (44) & $\mathrm{x}$ & $\mathrm{x}$ & & \\
\hline (45) & $\mathrm{x}$ & $\mathrm{x}$ & & \\
\hline (46) & $\mathrm{x}$ & $\mathrm{x}$ & & \\
\hline$(47)$ & $\mathrm{x}$ & & $\mathrm{x}$ & \\
\hline (48) & $\mathrm{x}$ & $x$ & $\mathrm{x}$ & \\
\hline (49) & $x$ & & $\mathrm{x}$ & \\
\hline$(50)$ & $\mathrm{x}$ & & $\mathrm{x}$ & \\
\hline (51) & $\mathrm{x}$ & & & $\mathrm{x}$ \\
\hline
\end{tabular}

$$
\begin{aligned}
& \forall X: \text { nat. } \forall Y: \text { nat. } \min (X, Y) \leqslant \max (X, Y), \\
& \forall X: \text { int. } \forall A: \text { list (int). } \forall B: \text { list (int). } X \in A \rightarrow X \in A \cup B, \\
& \forall X: \text { nat. half }(X+X)=X, \\
& \forall L: \text { list(int). } \forall P: \text { list (int). } \\
& \quad \text { rotate(length }(L), L<>P)=P<>L, \\
& \forall X: \text { nat. } \forall Y: \text { nat. } X \leqslant(X+Y), \\
& \forall X: \text { sexp. } \forall Y: \text { sexp. } \forall Z: \operatorname{sexp} . Z \in X \rightarrow X \in(X<>Y), \\
& \forall X: \text { int }^{+} . \exists L: \text { :list(prime). } \operatorname{prod}(L)=X .
\end{aligned}
$$

Table 1 shows the kinds of rippling used to prove each of these theorems. Note that CLAM can combine two or more kinds of rippling within a single proof.

To test the hypotheses, advanced in Section 2.5, that there is little or no search during rippling, we conducted the following experiment. Each wave-rule required during the proof of each of our 82 test theorems was made available to CLAM. A complete test run of the 82 theorems was made and any occurrences of branching were automatically recorded. There was no branching at all. That is, at each step, no more than one wave-rule met the rippling preconditions. We did not even detect the only kind of branching anticipated in Section 6.3, namely a choice between longitudinal 
and transverse wave-rules. It seems that, in practice, it is rare to have both kinds of wave-rule apply to the same expression.

\subsection{Case studies}

Below we give some case studies where rippling, as implemented in CLAM, can automatically prove theorems that other theorem provers cannot prove unaided. In each case we show how rippling is responsible for the improved performance. In comparing the performance of theorem provers we have tried to aid comparison between them by translating their formalisms into the one used in this paper. We claim that these translations do not violate the spirit of their proof attempts.

\subsubsection{Naive and tail-recursive list reversal}

Consider the theorem

$$
\forall L: l i s t(\text { nat }) . \forall M: l i s t(n a t), \operatorname{rev}(L)<>M=\operatorname{qrev}(L, M)
$$

discussed in Section 6.2. This example is based on one in [11] used to illustrate folding and unfolding. Unfolding is like rippling but restricted to the application of recursive rewrite rules and applied exhaustively. Folding is the inverse to unfolding, i.e. it is like rippling-in. In fact, the proof cannot be handled by folding and unfolding alone, because it requires the application of a non-recursive equation: the associativity of \langle\rangle . Darlington calls the associativity of $<>$ a law. Laws are applied by user intervention.

As we have seen in Section 6.2 , the associativity of $\langle>$ can be viewed as a wave-rule, in various ways. The appropriate way to use it is (usually uniquely) determined by the location of the wave-fronts in the induction conclusion. Thus rippling subsumes unfolding and is able to account for the application of a "law" that otherwise requires human intervention. In this case the annotation required on the associativity of $\langle>$ is as a transverse rule,

$$
\left(\underline{U}<>V{ }^{\uparrow}\right)<>W \Rightarrow U<>\left({ }^{V<>\underline{W}}{ }^{\dagger}\right),
$$

and it is used for the following piece of rippling-sideways:

$$
\begin{aligned}
& \left.(\underline{\underline{\operatorname{rev}(l)}<>(h:: n i l)}]^{\uparrow}\right)<>\lfloor m\rfloor=\operatorname{arev}\left(l,\left[h::\left.\underline{m}\right|^{\downarrow}\right\rfloor\right), \\
& \left.\operatorname{rev}(l)<>\left(\left\lfloor(h:: n i l)<>\left.\underline{m}\right|^{\downarrow}\right\rfloor\right)=\operatorname{arev}\left(l,|h:: \underline{m}|^{\downarrow}\right\rfloor\right) .
\end{aligned}
$$

Nqthm can prove this theorem automatically provided the associativity of $<>$ is available as a rewrite rule and that the user has oriented it in the appropriate direction. 


\subsubsection{Rotate length}

Consider the theorem

$$
\forall L: \operatorname{list}(\tau) . \forall A: \operatorname{list}(\tau) \text {, rotate }(\text { len }(L), L<>A)=A<>L \text {. }
$$

The function rotate takes a number, $n$, and a list, $l$, and returns a list identical to $l$ but with the first $n$ elements transported from the beginning to the end. Nqthm cannot prove this theorem. This is because it does not have the rippling-in tactic which follows rippling-sideways.

Both Nqthm and CLAM choose a " $h d::$ " induction ${ }^{10}$ on $L$. Since $A$ is also universally quantified, it is a sink, so the CLAM representation of the induction conclusion is

$$
\operatorname{rotate}\left(\operatorname{len}\left(\underline{h d:: \underline{t l}}^{\dagger}\right),\left(h_{h d:: \underline{t l}}^{\dagger}\right)<>\lfloor a\rfloor\right)=\lfloor a\rfloor<>\left(h d::\left.\underline{l l}\right|^{\dagger}\right) .
$$

The recursive definitions of $\langle>$, len, and rotate provide the following waverules:

$$
\begin{aligned}
& \left.(H d:: \underline{T l}]^{\uparrow}\right)<>L H d::(\underline{T l<>L}) \text {, } \\
& \left.\operatorname{len}\left(H d:: \underline{T l}{ }^{\dagger}\right) \Rightarrow s \underline{(\operatorname{len}(T l)}\right), \\
& L \neq \text { nil } \rightarrow \\
& \text { rotate }\left(\underline{s(\underline{N})}^{\dagger}, L\right) \\
& \Rightarrow \operatorname{rotate}\left(N, \operatorname{cdr}(\underline{L})<>(\operatorname{car}(\underline{L}):: n i l){ }^{\downarrow}\right) .
\end{aligned}
$$

CLAM can use these wave-rules for rippling; Nqthm can use the recursive definitions for symbolic evaluation. Thus both systems can use these definitions, together with some simplification, to rewrite the induction conclusion to

$$
\text { rotate } \left.(\text { len }(t l), \underline{(t l<>\lfloor a\rfloor})<>(h d:: n i l)^{\downarrow}\right)=\lfloor a\rfloor<>\left(\left.\underline{h d:: \underline{t l}}\right|^{\dagger}\right) .
$$

CLAM completes the rippling of the left-hand wave-front. It applies the associativity of $\langle>$ backwards as a longitudinal wave to ripple this wavefront towards its sink. Without a rather special-purpose wave-rule the righthand side wave-front is blocked.

This puts the induction conclusion in the form:

$$
\text { rotate } \left.\left(\operatorname{len}(t l), t l<>\left(\lfloor\underline{a}<>(h d:: n i l)]^{\dagger}\right\rfloor\right)\right)=\lfloor a\rfloor<>\left(\underline{h d:: \underline{t l}]^{\dagger}}\right) .
$$

\footnotetext{
${ }^{10}$ Actually Nqthm uses a destructor-style version of this, but it will aid the comparison and does not seriously distort the account, to pretend that it uses a constructor-style induction.
} 
Since the left-hand side is fully rippled, weak fertilization applies to give

$$
\left.\left.(\underline{\underline{a}<>(h d:: n i l)}]^{\dagger}\right]\right)<>t l=a<>(h d:: t l) .
$$

Rippling-in ${ }^{11}$ now uses the associativity of $<>$ as a transverse wave-rule to ripple the remaining wave-front out of the sink. This gives

$$
\left(\lfloor a\rfloor<>\left([(h d:: \text { nil })<>\underline{t l}]^{\downarrow}\right)=a<>(h d:: t l)\right.
$$

to which cancellation and simplification apply, yielding

$$
h d:: t l=h d:: t l \text {, }
$$

which is proved by the identity tactic.

Provided the associativity of $<>$ is available to Nqthm and has been appropriately oriented by the user, it will mimic the inwards ripple on the left-hand side. Although the left-hand side is now fully rippled, Nqthm is unable to apply weak fertilization because its logic does not permit any instantiation of $A$ except at the time of determining the induction rule. Nqthm then loses its way, overgeneralises and fails.

Nqthm lacks any heuristics corresponding to rippling-in or ripplingsideways and because of the limitations of its logic is unable to fully exploit sinks. If the transverse wave-rules used for rippling-sideways are based on recursive definitions (as is the case with the definition of rotate), then symbolic evaluation will mimic the effect of rippling-sideways. Also if it happens to have a rewrite rule oriented in the direction required for a particular application of rippling-out, -sideways or -in, then it will mimic these processes. But this is more a matter of luck than judgement; in many cases no happy coincidence will arise and Nqthm fail.

\section{Related work}

The use of sets of rewrite rules in theorem provers is commonplace. The most common usage is to apply them exhaustively to put expressions in a normal form. By "exhaustively" we mean that rules are applied until no further rule applications are possible. In contrast, we apply wave-rules selectively, that is as well as a rule being applicable some additional preconditions must be met, e.g. that wave-fronts can only be rippled sideways towards a sink, that anti-wave-fronts can only be introduced if they correspond to an existing wave-front.

Termination of the exhaustive application of a set of rewrite rules is an important property. This is usually proved by exhibiting a well-founded

\footnotetext{
${ }^{11}$ This is the kind of rippling-in using transverse wave-rules that was described in Section 6.3.
} 
ordering that is strictly decreased by each rule application. Since wave-rules are selectively applied, we are able to take account of the preconditions on the application when proving termination. For instance, in Appendix A we used the information that each rule application moves the wave-front along a fixed length path. This allows us to use wave-rules in either orientation without the risk of looping, something that is impossible with exhaustive application. The first exploitation, of which we are aware, of selective application of rewrite rules to prove termination, is in [10].

The decision as to which rules to include in a rewrite rule set and which way round to orient them is often partially determined by the termination ordering. It might also be determined automatically by a Knuth-Bendix process which is trying to create a confluent set [18]. Alternatively, it may simply be determined by the intuitions of the designer. In contrast, waverules are chosen and oriented automatically by CLAM on the basis of their syntactic structure, as given in Section 8. The same formula may used in several different ways, including both orientations. This technique for selection and orientation ensures that the rule set meets its specification of rippling wave-fronts. It also means that we can add to the rule set dynamically without risk of deviation from this specification, of non-termination or of combinatorial explosion.

The first documented use of rewrite rules based on recursive definitions was in [11], who called it unfolding. Exhaustive unfolding has since become standard practice in inductive theorem provers. Aubin [1] first pointed out how unfolding rippled (what we call) wave-fronts through the induction conclusion. He called this process rippling-out and exploited it in his generalisation-apart heuristic. In [4] we pointed out how Aubin's observation could be used to extend the class of rewrite rules used for rippling-out from recursive definitions to wave-rules. The present paper extends further our initial definition of wave-rules.

Building on the proposals in [4], Hutter has implemented a rippling-like heuristic in the INKA inductive theorem prover [15]. His implementation differs from the one described here in the following respects.

- His rewrite rule set contains any rule that moves wave-fronts ${ }^{12}$ within a skeleton. This is a larger rule set than our wave-rules, because it includes rules that move wave-fronts in directions other than out, sideways, or across.

- These rewrite rules are controlled by a series of strategies that correspond roughly to rippling-out, rippling-sideways, and rippling-across. There are minor differences that affect the relative power of the two implementations, but these are not significant.

${ }^{12} \mathrm{He}$ calls wave-fronts "contexts". 
Hutter independently invented the concept of rippling-across and possibly rippling-sideways. He also invented an interesting technique for the representation of wave-fronts. Each function has an additional argument that indicates whether it belongs to the skeleton or the wave-front. The rippling precondition of wave-front agreement is taken care of by an enhanced matcher. As currently realised this technique has some messy complications, e.g. each variable has to have an infinite number of aliases and the matcher is very complicated. We hope it may be possible to simplify the technique and adopt it in CLAM.

Rippling is the central idea behind our rational reconstruction of the induction heuristics embedded in the Nqthm theorem prover, [3]. In [7] we have shown how the method used by Boyer and Moore to select the best form of induction to prove a theorem can be rationally reconstructed as a look-ahead to see what form of induction maximises the chances that rippling will succeed. Similar remarks apply to the generalisation of a theorem prior to induction-an area on which we are currently working. Our understanding of the relation between rippling and induction has also enabled us to extend the Boyer-Moore heuristics beyond purely universal theorems to those involving existential quantifiers.

The "black box" nature of the Nqthm rewriting subsystem makes a direct comparison with rippling difficult. It is possible, however, to make some comparison based upon some general observations.

Firstly, the most general wave-rule schema presented in Section 8 demonstrates the uniformity of rippling. Such uniformity within the rewriting capabilities of Nqthm is less obvious. Nqthm has four classes of rules of which REWRITE and ELIM relate to rippling. The REWRITE class encapsulates both recursive definitions and the use of lemmas. The rules classified as ELIM are used to eliminate destructors from an induction hypothesis. In some sense the ELIM rules play the same role in Nqthm that creational wave-rules play in CIAM; they both replace destructor functions in the hypothesis with constructor functions in the conclusion.

Secondly, the merits of rippling with respect to controlling the application of rewrite rules is demonstrated by its success at handling potentially nonterminating sets of rewrites. In particular, by being more focused, rippling is able to fold as well as unfold recursive definitions. In contrast, Nqthm makes no provision for the folding of definitions and the onus of ensuring against the non-termination of rewriting is left to the user. The Nqthm user must decide how to orient lemmas as rewrite rules. Rippling determines the orientation of lemmas as wave-rules. The same lemma or definition can be used in both orientations by rippling without the risk of non-termination.

Thirdly, rippling was developed for a much stronger logic than Nqthm. As a consequence rippling can be seen as providing a more general control strategy. For instance, the notion of sinks cannot be exploited by Nathm 
since universal quantification is implicit within its logic.

\section{Limitations and further work}

The work described above has made considerable extensions to rippling. In this section we describe some ideas for further extensions and suggest some ways of applying rippling to solve other problems.

\subsection{Multi-coloured wave-holes}

When a wave-front contains multiple wave-holes it is often the case that each wave-hole corresponds to a different induction hypothesis. Consider, for instance, the induction conclusion (22) from Section 3.3:

$$
\max h t(\operatorname{tree}(\underline{l}, \underline{r})) \geqslant \min h t(\operatorname{tree}(\underline{l}, \underline{r})) .
$$

Each occurrence of tree contains two wave-holes. In both occurrences the first of these wave-holes corresponds to the induction hypothesis,

$$
\max \_h t(l) \geqslant \min \_h t(l),
$$

and the second to

$$
\max h t(r) \geqslant \min \_h t(r) .
$$

We can think of (22) as containing two distinct skeletons: one corresponding to each induction hypothesis. This correspondence could be inferred from the induction rule, just as the original wave annotations are. However, since it is not explicitly recorded, rippling can violate it, mixing the skeletons and leading to the failure of fertilization.

To see how a mixed skeleton might occur consider the induction conclusion

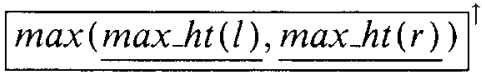

$$
\begin{aligned}
& \geqslant \min \left(\underline{\min \_h t(l)}, \underline{\min \_h t(r)}\right)^{\dagger},
\end{aligned}
$$

and suppose we have available the wave-rules,

$$
\begin{aligned}
& \underline{\max \left(\underline{U_{1}}, \underline{U_{2}}\right)}{ }^{\uparrow} \geqslant{\min \left(\underline{V_{1}}, \underline{V_{2}}\right)}^{\uparrow} \Rightarrow{\underline{U_{1} \geqslant V_{1}}} \wedge \underline{U_{2} \geqslant V_{2}}{ }^{\uparrow}, \\
& \left.\max \left(\underline{U_{1}}, \underline{U_{2}}\right){ }^{\uparrow} \geqslant \min ^{\left(V_{1}\right.}, \underline{V_{2}}\right){\underline{U_{1}}}^{\uparrow} V_{2} \wedge \underline{U_{2} \geqslant V_{1}}{ }^{\uparrow},
\end{aligned}
$$

where rule (54) is a commuted version of rule (53).

If wave-rule (54) is applied we get the induction conclusion

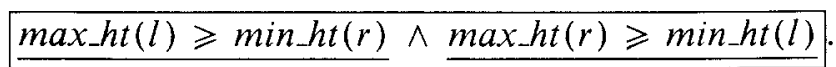


This contains two mixed skeletons. Neither of them will fertilize with the induction hypotheses. If wave-rule (53) is applied, we get the induction conclusion

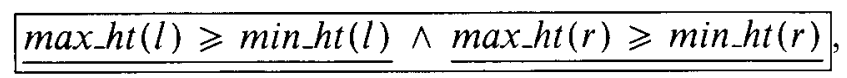

which fertilizes as required.

To prevent skeleton mixing we propose annotating multiple wave-holes in induction conclusions and wave-rules with colour labels and insisting that these labels match as a precondition of wave-rule application. Annotation with these labels can be readily automated. Induction conclusions will inherit their labels from induction rules. Induction rule and wave-rule colour labels can be calculated in a similar way to other annotations. In our example, induction conclusion (52) might have the annotation

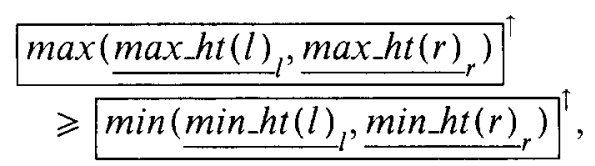

and the wave-rules (53) and (54) might have the annotation:

$$
\begin{aligned}
& \max \left({\underline{U_{1}}}_{X}, \underline{U_{2}}\right){ }^{\dagger} \geqslant \min \left(\underline{V_{1}}, \underline{V_{2}}\right){ }^{\uparrow} \\
& \Rightarrow \underline{U}_{1} \geqslant V_{1} \wedge \underline{U}_{2} \geqslant V_{2} Y \text {, } \\
& \max \left({\underline{U_{1}}}_{X},{\underline{U_{2}}}_{Y}\right) \min ^{\uparrow}\left(\underline{V_{1}}, \underline{V_{2}}\right){ }^{\uparrow} \\
& \Rightarrow \quad \underline{U_{1} \geqslant V_{2}} \wedge{\underline{U_{2}} \geqslant V_{1}}_{Y}^{\top} .
\end{aligned}
$$

Now wave-rule (54) does not apply to induction conclusion (52) because the colour labels will not match. Wave-rule (53) does apply, as required.

\subsection{Definition of wave-rules}

The definition of wave-rules has gradually grown more complex throughout this paper, culminating in the formats for generalised wave-rules in Section 8. These formats are hard to understand, sometimes ambiguous and awkward to work with. We are currently working towards an alternative definition based on meta-level properties. Under this new definition a waverule is an annotated rewrite rule that preserves its skeleton and decreases a measure.

The skeleton of one side of a wave-rule is the expression that lies outside the wave-front. Preserving it means that it must be the same on the left- and right-hand side of the wave-rule. The definition of skeleton is complicated by the existence of multiple wave-holes. The easiest way to solve this complication is to use the concept of coloured wave-hole (see Section 13.1 
above). The skeleton is then defined to be a set of expressions: one for each colour. For instance, the left- and right-hand skeletons of wave-rule (53),

$$
\begin{gathered}
\frac{\max \left(\underline{U_{1}},{\underline{U_{2}}}_{Y}\right)}{\uparrow} \geqslant \min \left(\underline{V_{1}}, \underline{V_{2}}\right) \\
\Rightarrow{\underline{U_{1}} \geqslant V_{1}}^{\uparrow} \wedge{\underline{U_{2}}}^{\uparrow}{\underline{V_{2}}}_{Y}
\end{gathered}
$$

are both $\left\{U_{1} \geqslant V_{1}, U_{2} \geqslant V_{2}\right\}$.

For a measure on sides of wave-rules we propose adapting the sequent measure defined in Appendix A. Decreasing this measure means that it should be smaller on the right-hand side than on the left. The use of the measure will make our termination proof redundant, since the use of a waverule will automatically decrease the measure of an induction conclusion and the measure is well-founded. The termination proof can be adapted to show that any rule meeting the various wave-rule formats in this paper will be a wave-rule under our new definition.

The new definition extends the concept of wave-rule. For instance, it allows the overlap of old and new wave-front positions, as in the transverse wave-rule:

$$
\left.\operatorname{rotate}\left(s(\underline{N}){ }^{\uparrow}, H d:: \underline{T l}\right]^{\uparrow}\right) \Rightarrow \operatorname{rotate}\left(N, \underline{T l<>(H d:: \text { nil })}{ }^{\downarrow}\right) .
$$

This does not fit the old format but it is desirable to regard it as a wave-rule.

This proposed new definition is reported as further work because it has not yet been implemented in CLAM and because we hope to further refine it. For instance, it is possible that the measure can be simplified by exploiting the concept of coloured wave-rule.

\subsection{Unblocking ripples}

Rippling can fail due to the lack of an appropriate wave-rule. We say that it is blocked. We are currently exploring various techniques for unblocking ripples. These mostly work by perturbing the induction conclusion in some way, but without disturbing the skeleton, in the hope that a wave-rule will apply to the perturbed expression. For instance, transverse wave-rules can be used in this way. Here is a simple, but rather artificial, example. Consider the blocked induction conclusion (25) from Section 4, which contained the subexpression $x+s(\underline{x}){ }^{\dagger}$, but where the wave-rule (26),

$$
U+s(\underline{V}){ }^{\uparrow} \Rightarrow \underline{(U+V)}{ }^{\uparrow},
$$

is not available. Suppose, however, that the rule

$$
U+s(\underline{V}){ }^{\uparrow} \Rightarrow \underline{\underline{U}}{ }^{\uparrow}+V
$$


is available. We can first apply rule (55) to unblock the ripple by moving the wave-front to the adjacent argument. This gives

$$
s(\underline{x})+x
$$

to which rule (4) applies to give

$$
s(\underline{x+x}){ }^{\dagger}
$$

as required. Blocked from moving outwards directly, the wave-front is first moved sideways around the blockage, and then outwards. Note the nonstandard annotation of rule (55), where the new wave-front is directed outwards rather than inwards. This reflects the non-standard use to which the rule is being put.

An alternative method of unblocking in the above example would be to apply the commutative law of + ,

$$
U+V \Rightarrow V+U
$$

It is often the case that commutative laws, and similar permutative rewrite rules, can be used to unblock ripples. Unfortunately, note that commutative laws are not wave-rules, and their uninhibited use could lead to looping. We are still studying how best to apply such permutative rules to unblock ripples. Possibilities include: (a) using them under loop-checking constraints, (b) building them into the unification algorithm, or (c) using them to generate permutated variants of wave-rules. Solution (c) is currently the favourite.

Another technique for unblocking ripples is to allow a blocked outwards wave-front with a single wave-hole containing a sink to reverse direction and be rippled in to this sink. This change of direction is a substitute for a missing transverse wave-rule. Instead of the wave-front going out, sideways and in, it goes out then in. Note that this is not just backtracking, because at least the later part of the path in will be different from the earlier part of the path out. For instance, consider the proof of

$$
\forall L: \text { list (nat). } \forall N: \text { nat. len }(L)+N=\operatorname{len}_{2}(L, N),
$$

where len is the naive list length function and $l e n_{2}$ is the tail-recursive version. Suppose we apply " $h:: l$ " induction and ripple out with the recursive definitions of len and + and ripple sideways with the recursive definition of $l e n_{2}$ :

$$
\left.s(\underline{\operatorname{len}(l)+\lfloor n\rfloor})]^{\dagger}=\operatorname{len}_{2}\left(l,[s(\underline{n})]^{\downarrow}\right\rfloor\right) .
$$

The ripple is now blocked. It can be unblocked by rippling-in with the wave-rule

$$
\left.U+s(\underline{V}){ }^{\uparrow} \Rightarrow \underline{\underline{U+V}}\right)^{\uparrow}
$$


applied right to left, to produce

$$
\operatorname{len}(l)+\left\lfloor[s(\underline{n})]^{\downarrow}\right\rfloor=\operatorname{len}_{2}\left(l, \mid\left[\left.s(\underline{n})\right|^{\downarrow}\right\rfloor\right)
$$

as required. This unblocking is a substitute for the (possibly missing) transverse wave-rule

$$
s(\underline{U}){ }^{\uparrow}+V \Rightarrow U+s(\underline{V})
$$

\subsection{Middle-out reasoning}

In [7] we describe how rippling can suggest which induction rule to use to prove a theorem. The idea is to look ahead to the step case and find an induction rule that would enable all the wave-fronts to be rippled at least once. This look-ahead is conducted as part of the preconditions of the induction tactic. We call it recursion analysis.

Recursion analysis is both inadequate and too inflexible: inadequate, because it only checks for the first step of rippling, and inflexible because it insists that all wave-fronts must be rippled at least once, when the best available induction rule may only be able to ripple some of them. In practice, we overcome these limitations with ad hoc devices. We are thus seeking an alternative mechanism that overcomes them in a principled way.

The mechanism we are currently exploring is to use meta-variables to postpone the choice of induction rule and allow it to be fixed by higher-order unification during rippling [5]. We call this middle-out reasoning because it allows us to postpone the beginning of a proof and tackle the middle first, allowing decisions made in the middle to influence the beginning. We can illustrate this with an example. Consider the theorem

$$
\forall X: n a t . \forall Y: n a t . e v e n(X) \wedge \text { even }(Y) \rightarrow \operatorname{even}(X+Y)
$$

Assume that the induction rule will introduce the induction terms $\chi(x)$ or $\gamma(y)$ or both. The step case will be:

$$
\begin{aligned}
& \operatorname{even}(x) \wedge \operatorname{even}(y) \rightarrow \operatorname{even}(x+y) \\
& \quad \vdash \operatorname{even}(\underline{\chi}(\underline{x})) \wedge \operatorname{even}(\gamma(y)) \rightarrow \operatorname{even}(\underline{y}(\underline{x})+\gamma(y)
\end{aligned}
$$

where we have used potential wave-fronts since we are not sure yet which of them will turn out to be required. The wave-rules (4) and (6) from Example 1 will both apply to the induction conclusion: the first in one way and the second in two. Each of these rule applications will eventually lead 
us to the same conclusion, but the most instructive is to apply rule (4), so let us start with that.

$$
\begin{aligned}
& \operatorname{even}(x) \wedge \operatorname{even}(y) \rightarrow \operatorname{even}(x+y)
\end{aligned}
$$

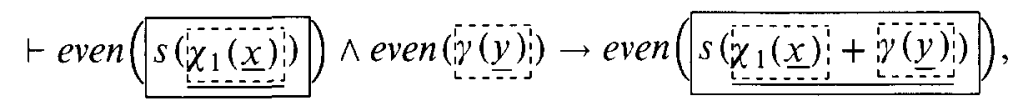

where $\chi(X)=s\left(\chi_{1}(X)\right)$. Now, since we always prefer to ripple real wavefronts before potential ones, the ripple with (6) is indicated, giving

$$
\begin{aligned}
& \operatorname{even}(x) \wedge \operatorname{even}(y) \rightarrow \operatorname{even}(x+y)
\end{aligned}
$$

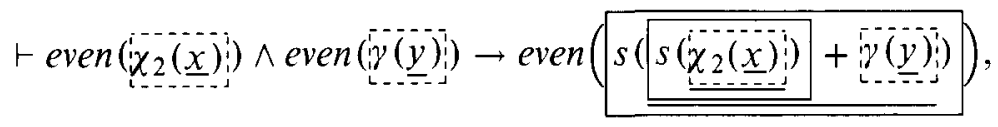

where $\chi_{1}(X)=s\left(\chi_{2}(X)\right)$. Another ripple with (4) is indicated, giving

$$
\begin{aligned}
& \operatorname{even}(x) \wedge \operatorname{even}(y) \rightarrow \operatorname{even}(x+y) \\
& \vdash \operatorname{even}\left(\chi_{2}(\underline{x})\right) \wedge \operatorname{even}(\hat{y}(\underline{y})) \rightarrow \operatorname{even}\left(s\left(s\left(\underline{\underline{x} 2(\underline{x})+y_{2}(\underline{y})}\right)\right),\right.
\end{aligned}
$$

and finally another with (6), giving

$$
\begin{aligned}
& \operatorname{even}(x) \wedge \operatorname{even}(y) \rightarrow \operatorname{even}(x+y) \\
& \vdash \operatorname{even}\left(\underline{\chi}_{2}(\underline{x})\right) \wedge \operatorname{even}\left(\underline { y } ( \underline { y } ) \rightarrow \operatorname { e v e n } \left(\underline{x}_{2}(\underline{x})+y_{1}(\underline{y})\right.\right.
\end{aligned}
$$

to which strong fertilization applies, instantiating $\chi_{2}(X)=X$ and $\gamma(Y)=$ $Y$. Thus $\chi(X)=s(s(X))$, i.e. a double induction on $X$ has been chosen, as required.

Middle-out reasoning can also be used to deal with existential quantifiers, and we are exploring this usage in parallel with our work on existential rippling. Meta-variables are used to postpone the commitment to an existential witness, then unification during subsequent rippling is used to retrospectively identify the witness. This use of meta-variables is fairly standard.

\subsection{Generalising-apart}

Aubin in [1] showed how to use recursion analysis to suggest generalisations of the theorem. He calls this mechanism generalisation-apart. Consider the following theorem

$$
\forall X: \text { nat. } X+(X+X)=(X+X)+X .
$$

This is a simple instance of the associativity of + . However, if we try to prove it using the obvious $s(X)$ induction the ripple gets blocked. Only the wave-fronts surrounding the first and fourth occurrences of $X$ can be fully rippled using wave-rule (4) from Example 1. No wave-rules apply at all to 
the third, fifth, and sixth occurrences and the second occurrence only ripples one level. The answer is to generalise the theorem to

$$
\forall X: \text { nat. } \forall Y: \text { nat. } X+(Y+Y)=(X+Y)+Y
$$

before trying $s(X)$ induction. Two of the occurrences of $X$ are generalised apart from the other four. This time wave-fronts only appear in positions where they can be fully rippled.

Recursion analysis can help suggest this generalisation. It locates those induction variable occurrences that can be rippled at least once. Unfortunately, this is not exactly what is wanted. It will locate the first, second, and fourth occurrences and suggest as generalisation the non-theorem:

$$
\forall X: \text { nat. } \forall Y: \text { nat. } X+(X+Y)=(X+Y)+Y \text {. }
$$

We want to reject the second occurrence on the grounds that it ripples once, but then gets blocked. So to reject this occurrence requires looking ahead more than one level. This suggests integrating generalisation suggestions into the middle-out reasoning process described in Section 13.4 above. Jane Hesketh is currently looking at this and other uses of middle-out reasoning for generalising theorems [14].

\subsection{Difference matching}

We have assumed, so far, that the wave annotations in the induction conclusion are initialised by the induction rule. However, it is desirable to have an independent method of introducing wave-fronts into an expression. One situation is when the induction rule suggests inappropriate wave-fronts and another is when rippling is used for non-inductive proofs. We illustrate each of these situations below.

A situation when rippling requires wave-fronts different to those initialised by the induction rule occurs in one of the proofs of the following simple example. Consider the theorem

$$
\forall N: \operatorname{nat} \text { even }(N) \vee \operatorname{even}(s(N)) \text {. }
$$

Recursion analysis uses wave-rule (6) from Example (1) to suggest an $s(s(N))$ induction, and this yields to the ind strat tactic with no problems. However, there is also a simple proof using $s(N)$ induction. The wave annotation suggested by this induction rule is

$$
\operatorname{even}(s(\underline{n})) \vee \operatorname{even}(s(\underline{s(\underline{n})})) \text {, }
$$

but this is no good. What is required instead is the wave annotation,

$$
\operatorname{even}(s(n)) \vee \operatorname{even}(s(s(\underline{n}))) \text {. }
$$


The commutativity of $\vee$ makes the skeleton of this induction conclusion match the induction hypothesis. One application of rule (6) then peters out the ripple and allows strong fertilization to finish the step case.

Summing series provides many examples of the use of rippling for noninductive proofs. ${ }^{13}$ For instance, consider the problem of finding a closed form solution to the series

$$
\sum_{i=0}^{n}(a \cdot i+b)
$$

where $a$ and $b$ are constants, using the standard forms,

$$
\begin{aligned}
& \sum_{I=0}^{N} K=(N+1) \cdot K, \\
& \sum_{I=0}^{N} I=\frac{1}{2} N \cdot(N+1) .
\end{aligned}
$$

and the wave-rules,

$$
\begin{aligned}
& \sum_{I=0}^{N} K \cdot \underline{U_{I}} \Rightarrow K \cdot \underline{\sum_{I=0}^{N} U_{I}}, \\
& \sum_{I=0}^{N} \underline{U}_{I}+\underline{V_{I}} \Rightarrow \sum_{I=0}^{N} U_{I}+\sum_{I=0}^{N} V_{I},
\end{aligned}
$$

where $K$ is a constant.

We can use rippling by treating the standard forms as induction hypotheses and using them to suggest wave-fronts in the problem series. Rippling-out these wave-fronts will allow weak fertilization to use the standard forms to replace occurrences of $\sum$ with closed form expressions. One of the standard forms that our problem series, (56), will match against is (57) above. This suggests the wave annotations

$$
\sum_{i=0}^{n} a \cdot i+\underline{b} .
$$

We can then ripple out with wave-rule (60) and weak fertilize with (57):

\footnotetext{
${ }^{13} \mathrm{We}$ are indebted to Toby Walsh for suggesting this application of rippling and providing the example below.
} 


$$
\begin{aligned}
\sum_{i=0}^{n} a \cdot i+\underline{b} & =\sum_{i=0}^{n} a \cdot i+\sum_{i=0}^{n} b \\
& =\sum_{i=0}^{n} a \cdot i+(n+1) \cdot b .
\end{aligned}
$$

We can then rematch the problem against the standard form (58) to suggest a new wave annotation, ripple out with wave-rule (59), and weak fertilize with (58):

$$
\begin{aligned}
\sum_{i=0}^{n} \underline{a} \underline{\underline{i}}+(n+1) \cdot b & =a \cdot \underline{\sum_{i=0}^{n} i}+(n+1) \cdot b \\
& =a \cdot \frac{1}{2} n \cdot(n+1)+(n+1) \cdot b .
\end{aligned}
$$

This technique has been implemented as an M.Sc. project by Alex Nunes and is reported in [19].

David Basin and Toby Walsh, in our research group, have designed a difference matching algorithm which inserts wave-fronts into goals [2]. It takes two expressions playing the role of induction hypothesis and induction conclusion, respectively, and identifies wave-fronts in the induction conclusion so that its skeleton matches the induction hypothesis. This algorithm can be used by a "stand-alone" rippling tactic which can find its own wave annotations and thus act independently of any induction rule. It has been implemented by Alex Nunes and is used in his series summing program to extend rippling in this way.

\section{Conclusion}

In this paper we have described the ripple tactic: the key search control heuristic for guiding inductive proofs. We started with the basic ripple-out tactic and then extended it in seven directions: multi-wave rippling, conditional rippling, rippling-in, rippling-sideways, rippling-across, and rippling under existential quantifiers. We then described a generalised ripple tactic which includes the first six of these extensions as special cases and allows hybrids between them.

Our extensions to rippling can be summarised as follows:

- multi-wave rippling is the generalisation of the form of wave-rules to simultaneous rippling of one or more wave-fronts each containing one or more wave-holes, finally matching with one or more induction hypotheses. This enables the use of strong fertilization and extends the proof plan to non-equational and non-equivalential theorems. 
- conditional rippling is the generalisation of rippling-out to conditional wave-rules. It uses covering sets of conditional rules to decide when to split into subcases.

- rippling-in uses wave-rules backwards either after weak fertilization or rippling-sideways, thus enabling these tactics to complete their task.

- rippling-sideways uses transverse wave-rules to move wave-fronts into sinks. It enables Oyster-CLAM to take advantage of free variables in the induction hypothesis.

- rippling-across is the adaptation of these constructor induction techniques to destructor induction.

- rippling under existential quantifiers is the adaptation of rippling to deal with existential quantifiers.

Rippling plays the central role in our proof plan for inductive proofs. As well as controlling the proof search in the step case of an induction it also helps to determine what inductive rule to use, how to generalise the theorem and what the existential witnesses should be. This proof plan and the rippling tactic have proved surprisingly successful. It has been successfully tested on 82 simple examples from the literature. No search is required. Our proof plan is much less sensitive than the Boyer-Moore theorem prover to the order in which theorems are presented; if an essential lemma is not available for rippling when required, an appropriate instance of it is sometimes proved "in-line".

However, our proof plan does not constitute a decision procedure for induction proofs, which is in any case an undecidable area. Nor does rippling always succeed in bridging the gap between induction hypothesis and induction conclusion. Rippling will block if the right wave-rule is not available. If it blocks on both sides of an equation, even weak fertilization will not apply. One way to proceed in such situations is shake the current goal into a form in which a wave-rule will apply. For instance, a wavefront might be moved sideways to a non-sink with a commutative law or transverse wave-rule. The non-standard use of wave-rules for unblocking has already been implemented in CLAM and other techniques are currently under investigation.

A general form of rippling has been implemented in Prolog in the OysterCLAM system. This generalised rippling includes as special cases: multi-wave rippling, conditional rippling, rippling-in, rippling-sideways, and ripplingacross. Existential rippling is partially implemented and under development.

\section{Appendix A. Termination}

The various forms of rippling direct and restrict the search for an inductive proof. The main purpose of using rippling is to avoid the combinatorial ex- 
plosion that is associated with exhaustive search techniques. It is, therefore, important to demonstrate that rippling always terminates. The termination of rippling seems plausible because it is always directed towards some finite objective, e.g. rippling a wave-front outwards until it is beached. Since expressions are of finite depth, the skeleton remains unchanged throughout rippling and each ripple moves the wave-front a non-zero distance through the skeleton in a fixed direction, so eventually no further movement will be possible. Unfortunately, this simple argument is complicated by the extensions to rippling described in this paper.

\section{A.1. Why proving termination is complicated}

Below we list these complicating factors.

(1) Creational rules create new wave-fronts as well as neutralising old ones. We must ensure that there is a limit to the new wave-fronts introduced.

(2) Rippling-in sends wave-fronts in the opposite direction to ripplingout. We must ensure that the two forms of rippling do not cause a loop.

(3) Wave-rules can duplicate parts of the skeleton, which also increases the number of wave-fronts. We must ensure that there is a limit to the new wave-fronts introduced.

(4) One wave-front can split into several, thus increasing the number of wave-fronts to be rippled. Each may be rippled along a different path. We must ensure that there is a limit to the new wave-fronts introduced.

(5) The path along which a wave-front is being rippled may be partially shared with another wave-front, and the two ripplings may interfere. We must ensure that this interference does not cause looping.

(6) If rippling fails to get a wave-front to its target, then it backs up and tries a different target. We must ensure that the search space is finite.

\section{A.2. The design of the well-founded measure}

To show that rippling always terminates we exhibit a well-founded measure that is strictly decreased by each application of a wave-rule. In designing this measure we address the above complications as follows:

- Problem (1) is solved by imposing a limit on the anti-wave-fronts that can be introduced. An anti-wave submeasure of wave-fronts in the induction hypothesis is defined and used as lexicographic component of the overall measure. This submeasure is decremented as these wavefronts are neutralised by the introduction of anti-wave-fronts in the 
induction conclusion. The submeasure is thus strictly monotonically decreasing and bounded below.

- Problem (2) is solved by treating inward directed wave-fronts and outward directed ones, separately. The overall measure is a lexicographically ordered triple of an anti-wave submeasure, an outwards submeasure and an inwards submeasure, in that order. So progress on the outward tree comes before progress on the inward one. Note that outward fronts can be re-labeled as inward, but not vice versa. Thus a wave-front can change direction at most once.

- Problem (4) is solved by attaching a weight to each wave-front such that if a compound front is split into several parts, then the weight of a compound front is equal to the combined weights of its parts. We will adopt the simple solution that the weight of a wave-front is the number of function applications in it. So, for instance, the weights of

$$
s(s(\underline{x})) \text { and } s(\underline{\underline{s(\underline{x})})}
$$

will both be 2-1 for each application of $s$. Note that weights are natural numbers.

- Problems (3) and (5) are solved by considering the overall effect of the wave-front application on the whole skeleton. Different techniques are used for the anti-wave, outwards, and inwards submeasures. For the anti-wave submeasure we use a natural number. For the outwards submeasure we use nested multisets where the nesting represents the structure of the skeleton and the possible positions within it for wavefronts. For the inwards submeasure we treat the skeleton as a tree labeled with (unnested) multisets of all the weights at that position in any of the duplicates. Sometimes distinct induction hypotheses have different skeletons. These skeletons differ only in the name of the induction variable. In the discussion below these skeletons are treated as identical.

- Problem (6) is solved because the search space is finite. There is only ever a finite branching rate because there are only a finite number of wave-rules. Each branch of the space is finite as shown by the wellfounded measure.

\section{A.3. The well-founded measure}

The measure attached to a sequent is a lexicographically ordered triple of the anti-wave, outwards, and inwards submeasures. The anti-wave submeasure is a natural number measuring the wave-fronts in the induction hypothesis. The outwards submeasure is a nested multiset of weights measuring the outwards directed wave-fronts. The inwards submeasure is the 
skeleton labeled with multisets of weights measuring the inwards directed wave-fronts.

We start by defining the anti-wave, outwards, inwards and overall (sub)measures. We represent nested and unnested multisets with set notation, e.g. $\{\ldots\}, \emptyset$, and $\cup$. We represent labeled trees by nested expressions in which the nodes are functions and the labels are superscripts on these functions. Finally, we define well-founded orders on the (sub)measures.

Definition A.1 (Anti-wave submeasure). $\|\Gamma\|^{-}$is the anti-wave submeasure of the sequent $\Gamma$. It is a natural number defined to be the sum of the weights of the wave-fronts in the induction hypothesis:

$$
\begin{aligned}
& \|\Gamma-\Delta\|^{-}=\|\Gamma\|^{-}, \\
& \left\|\eta\left(\mu_{1}, \ldots, \mu_{n}\right)\right\|^{-}=\sum_{i=1}^{n}\left\|\mu_{i}\right\|^{-}, \\
& \left\|\xi\left(\mu_{1}, \ldots, \mu_{n}\right)\right\|^{-}=1+\sum_{i=1}^{n}\left\|\mu_{i}\right\|^{-},
\end{aligned}
$$

where $\eta$ is an $n$-ary function in the skeleton and $\xi$ is an $n$-ary function not in the skeleton.

For example, the anti-wave submeasure of

$$
\begin{aligned}
& l \neq \text { nil, } \\
& \text { conscells }\left(\operatorname{car}(\underline{l}){ }^{\dagger}\right) \geqslant \operatorname{len}\left(\operatorname{car}(\underline{l}){ }^{\dagger}\right), \\
& \text { conscells }\left(\operatorname{cdr}(\underline{l}){ }^{\dagger}\right) \geqslant \operatorname{len}\left(\operatorname{cdr}(\underline{l}){ }^{\dagger}\right), \\
& \quad-\operatorname{conscells}(l) \geqslant \operatorname{len}(l)
\end{aligned}
$$

is 4 .

Definition A.2 (Outwards submeasure). $\|\Gamma\|^{\uparrow}$ is the outwards submeasure of the sequent $\Gamma$. It is a nested multiset defined recursively in two stages. We first define a labeled AND/OR tree, $\operatorname{tr}(\Gamma)$, that represents the duplicated skeleton with its weights, and then we define a nested multiset, $n m(\operatorname{tr}(\Gamma))$, from this AND/OR tree. Thus, $\|\Gamma\|^{\uparrow}=n m(\operatorname{tr}(\Gamma))$ :

$$
\begin{aligned}
& \operatorname{tr}(\Gamma \vdash \Delta)=\operatorname{tr}(\Delta), \\
& \operatorname{tr}\left(\eta\left(\mu_{1}, \ldots, \mu_{n}\right)\right)=\&^{0}\left(\eta\left(\operatorname{tr}\left(\mu_{1}\right), \ldots, \operatorname{tr}\left(\mu_{n}\right)\right)\right), \\
& \operatorname{tr}\left(\xi\left(\mu_{1}, \ldots, \mu_{n}\right)\right)=\&^{|\xi|+w_{1}+\cdots+w_{n}}\left(\operatorname{tr}\left(\mu_{1}^{1}\right), \ldots, \operatorname{tr}\left(\mu_{1}^{p_{1}}\right), \ldots,\right. \\
& \left.\operatorname{tr}\left(\mu_{n}^{1}\right), \ldots, \operatorname{tr}\left(\mu_{n}^{p_{n}}\right)\right),
\end{aligned}
$$

where: 
- $\eta$ is an $n$-ary function in the skeleton and $\xi$ is an $n$-ary function not in the skeleton,

- \& is a new variadic function,

- $|\xi|$ is 1 if $\xi$ is part of an outwards wave-front and 0 otherwise, and

- $\operatorname{tr}\left(\mu_{i}\right)=\&^{w_{i}}\left(\operatorname{tr}\left(\mu_{i}^{1}\right), \ldots, \operatorname{tr}\left(\mu_{i}^{p_{i}}\right)\right)$;

$$
\begin{aligned}
& n m\left(\eta\left(\mu_{1}, \ldots, \mu_{n}\right)\right)=\left\{n m\left(\mu_{1}\right), \ldots, n m\left(\mu_{n}\right)\right\} \\
& n m\left(\&^{w}\left(\mu_{1}, \ldots, \mu_{n}\right)\right)=\left\{w, n m\left(\mu_{1}\right), \ldots, n m\left(\mu_{n}\right)\right\} .
\end{aligned}
$$

For example, if $\Gamma$ is

$$
\begin{aligned}
& \ldots \vdash{s\left(\max \left(\max \_h t(l), \text { max_ht }(r)\right)\right)}^{\uparrow} \\
& \geqslant s\left(\min \left(\min \_h t(l), \underline{\left.\min \_h t(r)\right)}\right)\right),
\end{aligned}
$$

then $\operatorname{tr}(\Gamma)$ is

$$
\begin{aligned}
& \&^{0}\left(\geqslant\left(\&^{2}\left(\max \_h t\left(\&^{0}(l)\right), \max \_h t\left(\&^{0}(r)\right)\right),\right.\right. \\
& \left.\left.\&^{2}\left(\min \_h t\left(\&^{0}(l)\right), \min \_h t\left(\&^{0}(r)\right)\right)\right)\right),
\end{aligned}
$$

and $\|\Gamma\|^{\uparrow}$ is

$$
\{0,\{\{2,\{\{0, \emptyset\}\},\{\{0, \emptyset\}\}\},\{2,\{\{0, \emptyset\}\},\{\{0, \emptyset\}\}\}\}\}
$$

Definition A.3 (Inwards measure). $\|\Gamma\|^{\downarrow}$ is the inwards submeasure of the sequent $\Gamma$. It is a tree labeled with multisets and is defined recursively as follows.

$$
\begin{aligned}
& \|\Gamma \vdash \Delta\|^{\downarrow}=\|\Delta\|^{\downarrow}, \\
& \left\|\eta\left(\mu_{1}, \ldots, \mu_{n}\right)\right\|^{\downarrow}=\eta^{\{0\}}\left(\left\|\mu_{1}\right\|^{\downarrow}, \ldots,\left\|\mu_{n}\right\|^{\downarrow}\right), \\
& \left\|\xi\left(\eta_{1}, \ldots, \eta_{m}\right)\right\|^{\downarrow}=\eta^{\left\{\xi \mid+w: w \in \sigma_{1} \cup \ldots \cup \sigma_{m}\right\}}\left(u\left(\left\|\eta_{1}^{1}\right\|^{\downarrow}, \ldots,\left\|\eta_{1}^{m}\right\|^{\downarrow}\right), \ldots,\right. \\
& \left.u\left(\left\|\eta_{n}^{1}\right\|^{\downarrow}, \ldots,\left\|\eta_{n}^{m}\right\|^{\downarrow}\right)\right),
\end{aligned}
$$

where

- $u\left(\eta^{\sigma_{1}}\left(\left\|\eta_{1}^{1}\right\|^{\downarrow}, \ldots,\left\|\eta_{n}^{1}\right\|^{\downarrow}\right), \ldots, \eta^{\sigma_{i}}\left(\left\|\eta_{1}^{m}\right\|^{\downarrow}, \ldots,\left\|\eta_{n}^{m}\right\|^{\downarrow}\right)\right)$

$$
=\eta^{\sigma_{1} \cup \cdots \cup \sigma_{m}}\left(u\left(\left\|\eta_{1}^{1}\right\|^{\downarrow}, \ldots,\left\|\eta_{1}^{m}\right\|^{\downarrow}\right), \ldots, u\left(\left\|\eta_{n}^{1}\right\|^{\downarrow}, \ldots,\left\|\eta_{n}^{m}\right\|^{\downarrow}\right)\right),
$$

- $\eta$ is an $n$-ary function in the skeleton and $\xi$ is an $n$-ary function not in the skeleton,

- $|\xi|$ is 1 if $\xi$ is part of an inwards wave-front and 0 otherwise, and 
- $\left\|\eta_{i}\right\|^{\downarrow}=\eta^{\sigma_{i}}\left(\operatorname{tr}\left(\mu_{i}^{1}\right), \ldots,\left\|\mu_{i}^{n}\right\|^{\downarrow}\right)$; note that the function $\eta$ will be the same for all $1 \leqslant i \leqslant m$ up to renaming of induction variables, which we assume to take place as required.

For example,

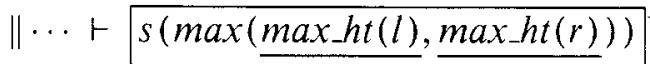

$$
\begin{aligned}
& \geqslant{\left.\overline{s\left(\min \left(\min \_h t(l), \min _{-} h t(r)\right)\right.}\right)}^{\downarrow} \|^{\downarrow}
\end{aligned}
$$

is

$$
\geqslant^{\emptyset}\left(\max _{-} h t^{\{2,2\}}\left(l^{\emptyset}\right), \min _{-} h t^{\{2,2\}}\left(l^{\emptyset}\right)\right) .
$$

Note that induction variable $r$ is renamed to $l$ in order to make all copies of the skeleton identical.

Definition A.4 (Sequent measure). $\|\Gamma\|$ is the measure of the sequent $\Gamma$. It is the triple of the anti-wave, outwards, and inwards submeasures, i.e.

$$
\|\Gamma\|=\left\langle\|\Gamma\|^{-},\|\Gamma\|^{\uparrow},\|\Gamma\|^{\downarrow}\right\rangle \text {. }
$$

Definition A.5 (Well-founded order). Let $\prec$ be an order on sequent measures defined as follows.

- The order, $\prec$, on the sequent measure, is a left/right lexicographic ordering. That is, $\left\langle a_{1}, o_{1}, i_{1}\right\rangle \prec\left\langle a_{2}, o_{2}, i_{2}\right\rangle$ iff either

- $a_{1}>a_{2}$, or

- $a_{1}=a_{2}$ and $o_{1} \ll^{*} o_{2}$, or

- $a_{1}=a_{2}, o_{1}=o_{2}$, and $i_{1} \prec_{i} i_{2}$.

- The order, $<$, on the anti-wave submeasure, is the standard less than order on the natural numbers.

- The order, $\ll^{*}$, on the outwards submeasure, is the nested multiset order [13], based on the numeric < order on the weights.

- The order, $\prec_{i}$, on the inwards submeasure, is a special root/leaves lexicographic ordering. Let $i_{1}$ and $i_{2}$ be trees and let $d$ be their upper difference set (defined below), consisting of a list of pairs of multisets of weights. $i_{1} \prec_{i} i_{2}$ iff $d$ is non-empty and for each pair $\left\langle\sigma_{1}, \sigma_{2}\right\rangle \in d$, $\sigma_{1} \ll \sigma_{2}$, where $\ll$ is the multiset order [13], based on the numeric < order on the weights.

Definition A.6 (Upper difference set). The difference set of two labeled trees of the same shape, $i_{1}$ and $i_{2}$, consists of all pairs of labels, $\left\langle\sigma_{1}, \sigma_{2}\right\rangle$, such that

- $\sigma_{1}$ is the label of node $n_{1}$ and $\sigma_{2}$ is the label of node $n_{2}$;

- $n_{1}$ and $n_{2}$ are corresponding nodes in $i_{1}$ and $i_{2}$, respectively;

- $\sigma_{1} \neq \sigma_{2}$; and 
- $n_{1}$ and $n_{2}$ are both maximal differing nodes, i.e. all corresponding nodes above them have equal labels.

By corresponding nodes we mean that each node is at the same position in its tree, where the two trees have the same shape.

Each of these constituent orders is well-founded. The well-foundedness of $<$ on the natural numbers and of lexicographic orderings on fixed length tuples are well known. The well-foundedness of $\ll$ and $\ll^{*}$ are both proved in [13]. The well-foundedness of $\prec_{i}$ on fixed shape trees follows by a similar argument for lexicographic orderings on fixed length tuples. So $\prec$ is well-founded.

\section{A.4. Rippling decreases the sequent measure}

We next show that our sequent measure is strictly decreased whenever a wave-rule is applied. To do this we will use the general format of wave-rules given in Section 8 . However, we will also have to use the preconditions of rippling, including the anti-wave, outward and inward annotations on wave-fronts. Since these differ for different directions of rippling, the proof will be in two cases: forwards applications of wave-rules and backwards applications of wave-rules.

\section{A.4.1. Forwards applications of wave-rules}

When rippling-out, rippling-sideways, rippling-across and hybrids of these, wave-rules are used forwards. The general format of wave-rules when used forwards is

$$
\begin{aligned}
& \text { Cond } \rightarrow \\
& \eta\left(\xi_{\xi_{1}\left(\mu_{1}^{1}, \ldots, \underline{\mu}_{1}^{p_{1}}\right)}^{\dagger}, \ldots, \xi_{\xi_{n}\left(\underline{\mu_{n}^{1}}, \ldots, \underline{\mu}_{n}^{p_{n}}\right)}^{\uparrow}, \nu_{1}, \ldots, \nu_{l}, \nu_{l+1}, \ldots, \nu_{m}\right)
\end{aligned}
$$

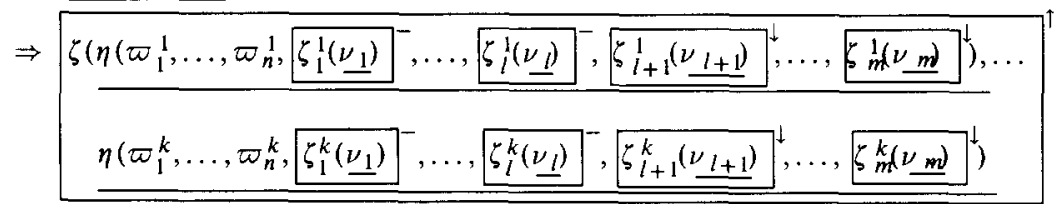

Note that $\eta$ is part of the skeleton.

Lemma A.7 (Decrease of measure during forward application). The sequent measure is strictly decreased by the forward application of a general wave-rule.

Proof. Note that either $l>0$ or $n>0$, i.e. if the rule is not purely creational, then it must have some wave-fronts on the left-hand side. 
Suppose that $l>0$, i.e. there are some anti-wave-fronts. It follows from the preconditions of applying generalised wave-rules (5) that at least one wavefront in the induction hypothesis must be neutralised. This will strictly decrease the anti-wave submeasure. Since the sequent measure is lexicographically ordered with the anti-wave submeasure first, it will also decrease strictly, even if its outwards and inwards submeasures increase.

Having dealt with the case $l>0$, we can assume that $l=0$ and $n>0$. Therefore, some outwards directed wave-fronts are rippled out or sideways. We will show the outwards submeasure is strictly decreased. Since $l=0$, the anti-wave submeasure is unchanged. The inwards submeasure may increase. Since the anti-wave, outwards, and inwards submeasures are lexicographically ordered the sequent measure is strictly decreased.

Consider the outwards submeasures, $\sigma$ and $\sigma^{\prime}$, of the before and after sequents, respectively. These two measures differ only in nested multisets corresponding to the $\eta$ function and some of its first $n$ arguments.

- The before measure, $\sigma$, contains a nested multiset, $\left\{w,\|\eta\|^{\top}, \ldots\right\}$, corresponding to the $\eta$ node. $\|\eta\|^{\top}$ contains a nested multiset, $\sigma_{i}$, for each of the $n$ outwards wave-fronts contained by $\eta$. The structure of $\sigma_{i}$ is: $\left\{w_{i},\left\|\mu_{i}^{1}\right\|^{\uparrow}, \ldots\left\|\mu_{i}^{p_{i}}\right\|^{\dagger}\right\}$

- The after measure, $\sigma^{\prime}$, is just like $\sigma$ except that an occurrence of $\left\{w,\|\eta\|^{\dagger}, \ldots\right\}$ is replaced by $\left\{w+|\zeta|,\left\|\eta_{1}\right\|^{\uparrow}, \ldots,\left\|\eta_{k}\right\|^{\dagger}, \ldots\right\}$.

We argue that this replacement causes a strict decrease in the outwards submeasure, since this new nested multiset is strictly smaller than the one it replaces. Each of its new elements is strictly smaller than $\|\eta\|^{\dagger}$.

- Firstly, $w+|\zeta| \ll^{*}\|\eta\|^{\uparrow}$, since $w+|\zeta|$ is a member of a base set, which is always smaller than any proper multiset under the nested multiset ordering, [13].

- Secondly, for $1 \leqslant j \leqslant k,\left\|\eta_{j}\right\|^{\uparrow} \ll^{*}\|\eta\|^{\uparrow}$, since each $\left\|\eta_{j}\right\|^{\uparrow}$ is just like $\|\eta\|^{\dagger}$ except that at least one of the nested multisets $\sigma_{i}$ it contains is replaced with a strictly smaller one, $\left\{w-\left|\xi_{i}\right|,\left\|\mu_{i}^{j^{\prime}}\right\|^{\top}\right\}$.

Therefore, $\sigma^{\prime} \ll^{*} \sigma$, i.e. the outwards submeasure is strictly decreased and, hence the sequent measure is strictly decreased.

\section{A.4.2. Backwards applications of wave-rules}

The general format of wave-rules when used for rippling-in is 
Cond $\rightarrow$

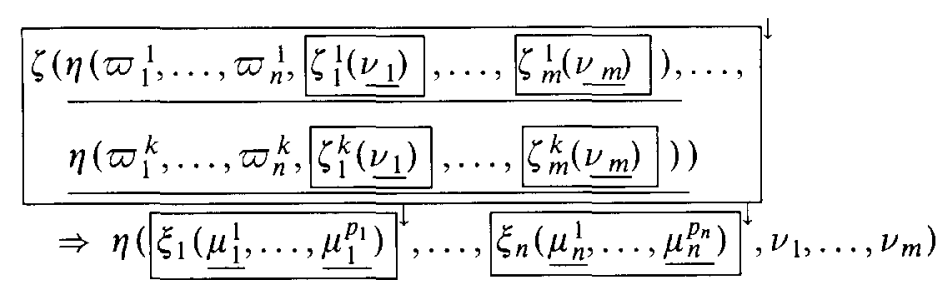

Note that $\zeta$ must not be empty, since purely transverse rules are not used for rippling-in.

Lemma A.8 (Decrease of measure during backward application). The sequent measure is strictly decreased by the backward application of a general wave-rule.

\section{Proof.}

(1) Note that the $\zeta_{i}^{j}$ wave-fronts can be annotated either outward or inward. The rule application removes them all. So if $m \neq 0$, then this will result in a strict decrease in the size of either the outwards or inwards submeasure or both.

(2) The inwards submeasures of the before and after formula differ only at the $\eta$ node and at some of the first $n$ of its arguments. Only the $\eta$ position is a maximal differing node, so the corresponding weight pair from this makes up the upper difference set. Since the $\zeta$ wave-front has been removed from this position, the weight at this node has strictly decreased. So the submeasure of the inwards tree has strictly decreased, whereas the submeasure of the outwards tree has either decreased or stayed the same.

Thus the overall measure is strictly decreased.

\section{A.4.3. The termination proof}

We can now put these two lemmas together to get the termination proof.

Theorem A.9 (Termination of rippling). The rippling tactic will terminate.

Proof. Rippling consists of repeated selective application of the general wave-rule format either forward or backward. By Lemma A.7 forward application decreases the sequent measure. By Lemma A. 8 backward application decreases the sequent measure. The sequent measure is well-founded. Therefore, rippling terminates.

Note that the argument only relied on the general format of wave-rules and the preconditions of rippling. Thus the termination proof holds for any 
set of wave-rules which fit the general format, even if some of the rules are reversals of others. No loop check is needed to ensure termination.

\section{Acknowledgements}

The research reported in this paper was supported by SERC grant GR/F/71799, an SERC Senior Fellowship to the first author and an SERC Postdoctoral Fellowship to the second author. We wish to thank our colleagues in the Edinburgh Mathematical Reasoning Group, three anonymous CADE-10 referees and two anonymous AIJ referees for feedback on this paper. An earlier, much shorter version of this paper appeared in the proceedings of CADE-10.

\section{References}

[1] R. Aubin, Some generalization heuristics in proofs by induction, in: G. Huet and G. Kahn, eds., Actes du Colloque Construction: Amélioration et vérification de Programmes (Institut de Recherche d'Informatique et d'Automatique, 1975).

[2] D. Basin and T. Walsh, Difference matching, in: D. Kapur, ed., 11th Conference on Automated Deduction, (1992), Lecture Notes in Artificial Intelligence 607 (Springer, Berlin, 1992) 295-309.

[3] R.S. Boyer and J.S. Moore, A computational logic (Academic Press, New York, 1979).

[4] A. Bundy, The use of explicit plans to guide inductive proofs, in: R. Lusk and R. Overbeek, eds., 9th Conference on Automated Deduction, (Springer, New York, 1988) 111-120; also longer version: DAI Research Paper No 349, University of Edinburgh, Edinburgh, Scotland.

[5] A. Bundy, A. Smaill and J. Hesketh, Turning eureka steps into calculations in automatic program synthesis, in: S.L.H. Clarke, ed., Proceedings UK IT 90, (1990) 221-226; also DAI Research Paper 448, University of Edinburgh, Edinburgh, Scotland.

[6] A. Bundy, F. van Harmelen, J. Hesketh and A. Smaill, Experiments with proof plans for induction, J. Autom. Reasoning 7 (1991) 303-324; also earlier version DAI Research Paper No 413, University of Edinburgh, Edinburgh, Scotland.

[7] A. Bundy, F. van Harmelen, J. Hesketh, A. Smaill and A. Stevens, A rational reconstruction and extension of recursion analysis, Proceedings IJCAI-89, Milan, Italy (1989) 359-365; also DAI Research Paper 419, University of Edinburgh, Edinburgh, Scotland.

[8] A. Bundy, F. van Harmelen, C. Horn and A. Smaill, The Oyster-Clam system, in: M.E. Stickel, ed., 10th International Conference on Automated Deduction, Lecture Notes in Artificial Intelligence 449 (Springer, New York, 1990) 647-648; also DAI Research Paper 507, University of Edinburgh, Edinburgh, Scotland.

[9] A. Bundy, F. van Harmelen, A. Smaill and A. Ireland, Extensions to the rippling-out tactic for guiding inductive proofs, in: M.E. Stickel, ed., 10th International Conference on Automated Deduction, Lecture Notes in Artificial Intelligence 449 (Springer, New York, 1990) 312-146; also DAI Research Paper 459, University of Edinburgh, Edinburgh, Scotland.

[10] A. Bundy and B. Welham, Using meta-level inference for selective application of multiple rewrite rule sets in algebraic manipulation, Artif. Intell. 16 (2) (1981) 189-212; also DAI Research Paper 121, University of Edinburgh, Edinburgh, Scotland. 
[11] R.M. Burstall and J. Darlington, A transformation system for developing recursive programs, J. ACM 24 (1) (1977) 44-67.

[12] R.L. Constable, S.F. Allen, H.M. Bromley et al., Implementing Mathematics with the Nuprl Proof Development System (Prentice Hall, Englewood Cliffs, NJ, 1986).

[13] N. Dershowitz and Z. Manna, Proving termination with multiset orderings, Comm. ACM 22 (8) (1979) 465-476.

[14] J.T. Hesketh, Using middle-out reasoning to guide inductive theorem proving, Unpublished Ph.D. Thesis, University of Edinburgh, Edinburgh, Scotland (1991).

[15] D. Hutter, Guiding inductive proofs, in: M.E. Stickel, ed., 10th International Conference on Automated Deduction, Lecture Notes in Artificial Intelligence 449 (Springer, New York, 1990) 147-161.

[16] T. Kanamori and H. Fujita, Formulation of induction formulas in verification of Prolog programs, in: J. Siekmann, ed., 8th Conference on Automated Deduction, Lecture Notes in Computer Science 230 (Springer, New York, 1986) 281-299.

[17] D. Kapur, ed., 11th Conference on Automated Deduction, (1992), Lecture Notes in Artificial Intelligence 607 (Springer, Berlin, 1992).

[18] D.E. Knuth and P.B. Bendix, Simple word problems in universal algebra, in: J. Leech, ed., Computational Problems in Abstract Algebra (Pergamon Press, Oxford, 1970) 263297.

[19] T. Walsh, A. Nunes and A. Bundy, The use of proof plans to sum series, in: D. Kapur, ed., 11th Conference on Automated Deduction, (1992), Lecture Notes in Artificial Intelligence 607 (Springer, Berlin, 1992) 325-339. 